\title{
The Evolution of Ultraviolet Emission Lines From Circumstellar Material Surrounding SN 1987A
}

\section{Citation}

Sonneborn, George, Claes Fransson, Peter Lundqvist, Angelo Cassatella, Roberto Gilmozzi, Robert P. Kirshner, Nino Panagia, and Willem Wamsteker. 1997. "The Evolution of Ultraviolet Emission Lines From Circumstellar Material Surrounding SN 1987A." The Astrophysical Journal 477 (2): 848-64. https://doi.org/10.1086/303720.

\section{Permanent link}

http://nrs.harvard.edu/urn-3:HUL.InstRepos:41399821

\section{Terms of Use}

This article was downloaded from Harvard University's DASH repository, and is made available under the terms and conditions applicable to Other Posted Material, as set forth at http:// nrs.harvard.edu/urn-3:HUL.InstRepos:dash.current.terms-of-use\#LAA

\section{Share Your Story}

The Harvard community has made this article openly available.

Please share how this access benefits you. Submit a story.

Accessibility 
The Astrophysical Journal, 477:848-864, 1997 March 10

(C) 1997. The American Astronomical Society. All rights reserved. Printed in U.S.A.

\title{
THE EVOLUTION OF ULTRAVIOLET EMISSION LINES FROM CIRCUMSTELLAR MATERIAL SURROUNDING SN 1987A
}

\author{
George Sonneborn, ${ }^{1,2}$ Claes Fransson, ${ }^{3}$ Peter Lundqvist, ${ }^{3}$ Angelo Cassatella,,${ }^{4}$ Roberto Gilmozzi, ${ }^{5}$ \\ Robert P. Kirshner, ${ }^{6}$ Nino PANAGIA, ${ }^{7,8}$ AND Willem WamsteKeR ${ }^{9}$ \\ Received 1996 February 26; accepted 1996 October 1
}

\begin{abstract}
The presence of narrow high-temperature emission lines from nitrogen-rich gas close to SN 1987A has been a principal observational constraint on the evolutionary status of the supernova's progenitor. A new analysis of the complete 5 year set of low- and high-resolution IUE ultraviolet spectra of SN 1987A (1987.2-1992.3) provides fluxes for the $\mathrm{N} v \lambda 1240, \mathrm{~N}$ IV] $\lambda 1486, \mathrm{He}$ II $\lambda 1640, \mathrm{O}$ III] $\lambda 1665, \mathrm{~N}$ III] $\lambda 1751$, and $\mathrm{C}$ III] $\lambda 1908$ lines with significantly reduced random and systematic errors and reveals significant short-term fluctuations in the light curves. The $\mathrm{N} \mathrm{v,} \mathrm{N} \mathrm{IV],} \mathrm{and} \mathrm{N} \mathrm{III]} \mathrm{lines} \mathrm{turn} \mathrm{on} \mathrm{sequentially} \mathrm{over}$ 15-20 days and show a progression from high to low ionization potential, implying an ionization gradient in the emitting region. The line emission turns on suddenly at $83 \pm 4$ days after the explosion, as defined by $\mathrm{N}$ IV]. The $\mathrm{N}$ III] line reaches peak luminosity at $399 \pm 15$ days. A ring radius of $(6.24 \pm 0.20) \times 10^{17} \mathrm{~cm}$ and inclination of $41.0 \pm 3.9$ is derived from these times, assuming a circular ring. The probable role of resonant scattering in the $\mathrm{N} v$ light curve introduces systematic errors that leads us to exclude this line from the timing analysis. A new nebular analysis yields improved CNO abundance ratios of $\mathrm{N} / \mathrm{C}=6.1 \pm 1.1$ and $\mathrm{N} / \mathrm{O}=1.7 \pm 0.5$, confirming the nitrogen enrichment found in our previous paper. From the late-time behavior of the light curves we find that the emission originates from progressively lower density gas and that the emitting region has a multicomponent density structure. We estimate the emitting mass near maximum ( $\sim 400$ days) to be $\sim 4.7 \times 10^{-2} M_{\odot}$, assuming a filling factor of unity and an electron density of $2.6 \times 10^{4} \mathrm{~cm}^{-3}$. These results are discussed in the context of current models for the emission and hydrodynamics of the ring.
\end{abstract}

Subject headings: circumstellar matter - supernovae: individual (SN 1987A) - supernova remnants ultraviolet: ISM

\section{INTRODUCTION}

Narrow emission lines from SN 1987A were first seen with the International Ultraviolet Explorer (IUE) satellite in 1987 May (Fransson et al. 1989, hereafter Paper I). The lines detected were $\mathrm{N} v \lambda \lambda 1239,1243, \mathrm{~N}$ Iv] $\lambda 1486, \mathrm{He}$ II $\lambda 1640$, O III] $\lambda \lambda 1661,1667, \mathrm{~N}$ III] $\lambda \lambda 1747-1754$, and C III] $\lambda \lambda 1907$, 1909 . The absence of the usually strong $\mathrm{Si}$ IV $\lambda 1400$, and C IV $\lambda 1550$ resonance lines can be attributed to absorption of these transitions by the same ions in hot interstellar gas in the LMC. The presence of N v in the SN 1987A spectra, on the other hand, is in fact an indication that there is a negligible column density of interstellar $\mathrm{N} v$ in the LMC. A remarkable feature of the early observations of SN 1987A was that the $\mathrm{N} \mathrm{III],} \mathrm{N} \mathrm{v}$, and $\mathrm{C} \mathrm{III]} \mathrm{lines} \mathrm{increased} \mathrm{in} \mathrm{flux}$ linearly with time. The $\mathrm{He}$ II, N IV], and $\mathrm{O}$ III] lines were

\footnotetext{
${ }^{1}$ Laboratory for Astronomy and Solar Physics, Code 681, NASA/ Goddard Space Flight Center, Greenbelt, MD 20771; sonneborn@fornax.gsfc.nasa.gov.

${ }^{2}$ Institut d'Astrophysique de Paris, 98bis boulevard Arago, 75014 Paris, France.

${ }^{3}$ Stockholm Observatory, S-133 36 Saltsjöbaden, Sweden; claes@astro.su.se,peter@astro.su.se.

${ }^{4}$ Istituto di Astrofisica Spaziale, CNR, CP 67, I-00044 Frascati, Italy; ac@vilspa.esa.es.

${ }_{5}$ European Southern Observatory, Karl-Schwarzschild Str. 2, 85748 Garching bei München, Germany; rgilmozz@eso.org.

${ }^{6}$ Center for Astrophysics, 60 Garden Street, Cambridge, MA 02138; kirshner@cfa.harvard.edu.

7 Space Telescope Science Institute, 3700 San Martin Drive, Baltimore, MD 21218; panagia@stsci.edu.

${ }^{8}$ Affiliated with the Astrophysics Division, Space Sciences Department of ESA.

${ }^{9}$ IUE Observatory, ESA-VILSPA, Casilla 50727, E-28080 Madrid, Spain;ww@vilspa.esa.es.
}

weak, and it was difficult to say anything conclusive about their light curves. The increase in the fluxes stopped just after the last observations discussed in Paper I, 400 days after explosion. Subsequent observations showed that the $\mathrm{C}$ III], N III], and $\mathrm{N} v$ lines reached a maximum $\sim 405$ days after the explosion (Sonneborn et al. 1988). After this epoch the observations reported in this paper show a steady decay of the lines. Earlier versions of the IUE emission-line light curves, but not the new measurements discussed in this paper, have been presented by us in several papers (Sonneborn et al. 1990; Panagia et al. 1991; Sonneborn 1991) and an independent analysis by Sanz Fernández de Córdoba (1993). Preliminary versions of some of the new light curves have been shown by Fransson \& Sonneborn (1994) and Plait et al. (1995). In a complementary paper to this one, Pun et al. (1995) analyzed the UV spectrophotometric evolution of the SN 1987A debris from day 1.6 to 1567 in IUE spectra covering $1150-3300 \AA$. They found good agreement with UV continuum observations of SN 1987A with Hubble Space Telescope (HST) and groundbased photometry where they overlap.

The presence and temporal development of these highly ionized lines have been explained as a result of recombination, cooling, and light echo effects (Lundqvist \& Fransson 1987; Chevalier 1988; Paper I; Lundqvist \& Fransson 1991, hereafter LF91). The initial pulse of soft X-rays accompanying the breakout of the shock wave ionizes the circumstellar gas expelled by the progenitor star. As the outburst evolves, an increasingly large volume of the ionized gas becomes visible because of light travel time effects. If the recombination time is long compared to the epoch of observation, the observed flux is expected to grow 
in proportion to the emitting volume within the light echo paraboloid. For a nonrecombining ion emitting at constant temperature this leads to a flux increasing monotonically with time, until the whole structure is within the light echo paraboloid. In reality, the temperature and ionization decrease with time, causing the evolution to become highly complicated. Detailed modeling of the early line emission showed that the peak radiation temperature at the time of the breakout was between $4 \times 10^{5}$ and $8 \times 10^{5} \mathrm{~K}$ (Fransson \& Lundqvist 1989; Lundqvist 1991). A subsequent analysis, taking the ring geometry into account, resulted in a peak radiation temperature in excess of $10^{6} \mathrm{~K}$ (Lundqvist \& Fransson 1996, hereafter LF96). These emission-line observations therefore provide important information about the first moments of the supernova explosion.

Another important piece of information was the large nitrogen enrichment derived from the emission-line ratios, primarily the $\mathrm{N} \mathrm{III]/C} \mathrm{III]} \mathrm{and} \mathrm{N} \mathrm{III]/O} \mathrm{III].} \mathrm{Abundance}$ ratios of $\mathrm{N} / \mathrm{C}=7.8 \pm 4$ and $\mathrm{N} / \mathrm{O}=1.6 \pm 0.8$, by number, found in Paper I strongly suggest CNO enriched gas. This has proven to be one of the main observational constraints on the progenitor evolution (e.g., Saio, Nomoto, \& Kato 1988; Woosley 1988; Weiss 1991; Podsiadlowski 1992), implying that the progenitor was in a post He-core burning phase at the time of the explosion.

After the discovery of the UV emission lines, circumstellar emission has also been observed at other wavelengths. In the optical a large number of lines from both medium ionized species and low-ionization ions have been detected (Allen, Meikle, \& Spyromilio 1989; Wampler \& Richichi 1989; Wampler, Richichi, \& Baade 1989; Crotts \& Kunkel 1991; Kahn \& Duerbeck 1991; Meikle et al. 1991; Menzies 1991; Wang 1991; Cumming 1994). Extended looplike structures surrounding SN 1987A were first imaged in $\mathrm{H} \alpha+[\mathrm{N} \mathrm{II}]$ and [O III] emission by Crotts, Kunkel, \& McCarthy (1989). Even in these early images the inner emission region appeared to be a hollow oval. These structures were also seen in optical continuum, indicating the presence of time-delayed reflections by dust. Imaging with the New Technology Telescope resolved the inner emission region of radius $\sim 1^{\prime \prime}$ and several outer structures (Wampler et al. 1990; Wang \& Wampler 1992). HST images in [O III] $\lambda \lambda 4959,5007$ (Jakobsen et al. 1991, 1994; Plait et al. 1995) revealed the now well-known thin ring geometry of the inner region, rather than a spherical shell as had been previously hypothesized. Further HST observations showed that the outer structures are concentrated mainly to two off-center rings, which extend $\sim 2$ ".5 to the north and south of the supernova (Burrows et al. 1995). Crotts, Kunkel, \& Heathcote (1995) show that the toroidal geometry extends also to regions of the circumstellar medium exterior to the ring and that there may be some connection between the inner and outer rings. They also find that the inner ring is circular to a high degree of accuracy. In addition to the geometry, the dynamics and origin of the inner ring is severely constrained by the emission-line widths. In Paper I we showed that the C III] $\lambda \lambda 1907,1909$ lines were very narrow (FWHM $<30 \mathrm{~km} \mathrm{~s}^{-1}$ ) and unshifted with respect to the LMC ISM toward the supernova $(v=285 \pm 6 \mathrm{~km}$ $\mathrm{s}^{-1}$ ). Crotts \& Heathcote (1991) and Cumming et al. (1997) found that the ring expands with a velocity of $10.3 \pm 0.4 \mathrm{~km}$ $\mathrm{s}^{-1}$. They, as well as Wood \& Faulkner (1987) and Meikle et al. (1991), also find a velocity gradient over the nebula, which is a strong indication of a ring geometry rather than a spherical shell.

Prompt radio emission from SN 1987A, the result of shock interaction with the progenitor's stellar wind close to the photosphere, was observed during the first few days after explosion (Turtle et al. 1987). This emission decayed away after the first week. However, around day 1200 there was a revival of the radio emission and a steady increase in the flux was observed (Staveley-Smith et al. 1992, 1993) that continued through day 2750 (Ball et al. 1995). Chevalier (1992) and Duffy, Ball, \& Kirk (1995) attribute the resurgence of the radio emission from SN 1987A to the interaction of the supernova ejecta with the density enhancement expected at the position of the termination shock in the blue supergiant wind of the progenitor. Further evidence of circumstellar interaction is the increasing latetime X-ray emission observed by ROSAT (Beuermann, Brandt, \& Pietsch 1994; Gorenstein, Hughes, \& Tucker 1994; Hasinger, Aschenbach, \& Trümper 1996). Chevalier \& Dwarkadas (1995) point out that the density of the shocked blue supergiant wind is insufficient to explain the late radio and X-ray fluxes as well as the apparent slowing down of the supernova shock (Staveley-Smith et al. 1993). They propose that the supernova shock is now moving into the denser $\left(n_{e} \sim 10^{2} \mathrm{~cm}^{-3}\right)$ shocked red supergiant wind that was ionized by the progenitor.

In this paper we discuss the complete set of UV emission lines in SN 1987A derived from IUE low- and highdispersion SWP spectra from the explosion up to 1992 April 20 (day 1882). A new method of analysis has allowed us to reduce systematic and random errors in the line measurements compared to Paper I and the light curves of Panagia et al. (1991) and Sanz Fernández de Córdoba (1993). The evolution of the line fluxes, leading to times for turn-on and maximum of the line emission, enable us to determine the absolute dimensions of the emitting gas independent of the imaging observations. The implications of these results in connection with ground-based and HST observations are discussed. In $\S 2$ we discuss the observations, in $\S 3$ the measurements of the line fluxes, and in $\S 4$ the main observational results are given. The paper concludes with a discussion $(\S 5)$ of ring geometry, abundances, density, emitting mass, and comparison with existing ring models in light of the observational data.

\section{OBSERVATIONS}

The ultraviolet spectra presented in this paper were obtained with the IUE SWP camera between 1987 March 14 and 1992 April 20. All spectra were taken in the $10^{\prime \prime} \times 20^{\prime \prime}$ short wavelength large aperture (SWLA), the minor axis of which is approximately parallel with the spectrograph's dispersion direction. The unique characteristics of the SN 1987A field demanded special care during the acquisition process to ensure accurate target centering in the SWLA, as discussed below. The observations were carried out at both IUE Observatories, located at NASA/ Goddard Space Flight Center and ESA's Villafranca ground station (VILSPA). The SWP image number, time of observation, and exposure duration for each low-dispersion spectrum are listed in Table 1. The observation date refers to the starting time of the exposure, expressed in days after outburst. The outburst time is assumed to be that of the IMB neutrino detection (1987 February 23.316 UT, Bionta et al. 1987). 
TABLE 1

EMISSION-LINE FLUXES FOR SN 1987A CIRCUMSTELLAR RING

\begin{tabular}{|c|c|c|c|c|c|c|c|c|c|}
\hline \multirow[b]{2}{*}{ SWP $^{\mathrm{a}}$} & \multirow[b]{2}{*}{$\begin{array}{l}\text { DATE }^{b} \\
\text { (days) }\end{array}$} & \multirow[b]{2}{*}{$\begin{array}{c}t_{\text {expo }}^{\mathrm{c}} \\
\text { (minutes) }\end{array}$} & \multirow[b]{2}{*}{$\begin{array}{c}v_{\text {corr }}^{\mathrm{d}} \\
\left(\mathrm{km} \mathrm{s}^{-1}\right)\end{array}$} & \multicolumn{6}{|c|}{$\begin{array}{l}\text { INTEGRATED FLUX } \\
\left(10^{-15} \mathrm{erg} \mathrm{cm}^{-2} \mathrm{~s}^{-1}\right)\end{array}$} \\
\hline & & & & $\begin{array}{l}\mathrm{N} v \\
1240\end{array}$ & $\begin{array}{l}\text { N IV] } \\
1486\end{array}$ & $\begin{array}{l}\text { He II } \\
1640\end{array}$ & $\begin{array}{l}\mathrm{O} \text { III] } \\
1666\end{array}$ & $\begin{array}{l}\mathrm{N} \text { iII] } \\
1750\end{array}$ & $\begin{array}{l}\text { C IIII] } \\
1908\end{array}$ \\
\hline $30440 \ldots \ldots \ldots$ & 11.3 & 300 & -257 & 5 & 13 & 2 & $\ldots$ & 1 & $\ldots$ \\
\hline $30472 \ldots \ldots \ldots$ & 14.0 & 180 & 46 & 14 & -7 & 14 & $\ldots$ & 4 & 1 \\
\hline $30512 \ldots \ldots \ldots$ & 18.3 & 240 & -46 & 2 & -9 & -1 & 17 & $\ldots$ & 6 \\
\hline $30522 \ldots \ldots \ldots$ & 19.1 & 181 & 591 & 11 & 4 & -2 & 7 & 16 & -4 \\
\hline $30547 \ldots \ldots \ldots$ & 21.3 & 240 & 111 & 6 & $\ldots$ & -2 & 17 & 10 & 5 \\
\hline $30592 \ldots \ldots \ldots$ & 27.3 & 300 & -125 & 3 & 7 & 19 & -2 & 6 & -5 \\
\hline $30637 \ldots \ldots \ldots$ & 33.1 & 185 & -106 & 2 & 10 & 18 & -5 & 4 & 5 \\
\hline $30743 \ldots \ldots \ldots$ & 44.2 & 225 & 53 & 4 & 21 & 4 & 6 & 1 & 2 \\
\hline $30907 \ldots \ldots \ldots$ & 70.2 & 230 & 164 & 7 & 4 & 26 & 19 & 14 & 7 \\
\hline $30974 \ldots \ldots \ldots$ & 80.6 & 120 & 303 & 55 & 2 & 49 & $\ldots$ & 12 & 11 \\
\hline $31000 \ldots \ldots \ldots$ & 85.2 & 180 & -37 & 69 & 45 & 70 & 12 & 17 & 8 \\
\hline $31040 \ldots \ldots \ldots$ & 90.9 & 195 & 233 & 64 & 114 & 33 & 35 & 29 & 17 \\
\hline $31064 \ldots \ldots \ldots$ & 96.3 & 180 & 199 & 84 & 101 & 74 & 12 & 76 & 6 \\
\hline $31125 \ldots \ldots \ldots$ & 105.8 & 200 & -7 & 105 & 77 & 55 & 27 & 117 & 13 \\
\hline $31132 \ldots \ldots \ldots$ & 107.8 & 195 & 42 & 92 & 95 & 67 & 42 & 109 & 6 \\
\hline $31154 \ldots \ldots \ldots$ & 110.8 & 180 & -154 & 144 & 107 & 54 & 68 & 113 & 27 \\
\hline $31166 \ldots \ldots \ldots$ & 113.0 & 180 & -83 & 107 & 108 & 70 & 50 & 88 & 29 \\
\hline $31245 \ldots \ldots \ldots$ & 121.7 & 260 & 8 & 98 & 155 & 43 & 66 & 90 & 91 \\
\hline $31273 \ldots \ldots \ldots$ & 128.0 & 240 & 111 & 98 & 131 & 75 & 66 & 191 & 38 \\
\hline $31319 \ldots \ldots \ldots$ & 138.5 & 240 & 7 & 115 & 163 & 92 & 75 & 170 & 70 \\
\hline $31371 \ldots \ldots \ldots$ & 147.0 & 240 & 130 & 159 & 117 & 94 & 64 & 215 & 78 \\
\hline $31420 \ldots \ldots \ldots$ & 154.5 & 240 & 280 & 163 & 141 & 116 & 94 & 207 & 72 \\
\hline $31462 \ldots \ldots \ldots$ & 160.9 & 240 & 122 & 140 & 139 & 85 & 139 & 190 & 53 \\
\hline $31534 \ldots \ldots \ldots$ & 168.5 & 220 & 396 & 150 & 117 & 78 & 88 & 265 & 88 \\
\hline $31592 \ldots \ldots \ldots$ & 177.7 & 240 & 243 & 186 & 136 & 93 & 84 & 183 & $\mathrm{X}^{\mathrm{e}}$ \\
\hline $31651 \ldots \ldots \ldots$ & 185.4 & 210 & -282 & 146 & 92 & 95 & 141 & 222 & 94 \\
\hline $31676 \ldots \ldots \ldots$ & 188.8 & 240 & -119 & 147 & 157 & 60 & 130 & 233 & $\mathrm{X}$ \\
\hline $31819 / 18 \ldots \ldots$. & 198.9 & $240 / 90$ & $-32 / 173$ & 163 & 186 & 67 & 108 & 302 & 106 \\
\hline $31893 \ldots \ldots \ldots$ & 209.6 & 200 & -104 & 175 & 181 & 75 & 93 & 268 & 110 \\
\hline $31954 \ldots \ldots \ldots$ & 220.3 & 200 & -926 & 131 & 148 & 74 & 99 & 265 & 101 \\
\hline 32030/31 ...... & 227.7 & $240 / 90$ & $-142 /-34$ & 139 & 177 & 110 & 155 & 389 & 101 \\
\hline $32168 \ldots \ldots \ldots$ & 245.5 & 155 & 520 & & $\ldots$ & $\ldots$ & $\ldots$ & 343 & 102 \\
\hline $32219 / 20 \ldots \ldots \ldots$ & 252.6 & $240 / 80$ & $-352 /-370$ & 176 & 126 & 90 & 85 & 323 & 113 \\
\hline $32314 \ldots \ldots \ldots$ & 263.4 & 235 & 46 & 215 & 161 & 93 & 94 & $\mathrm{X}$ & $\mathrm{X}$ \\
\hline $32395 \ldots \ldots \ldots$ & 275.8 & 90 & -500 & $\ldots$ & $\ldots$ & $\ldots$ & $\ldots$ & 321 & 135 \\
\hline $32404 \ldots \ldots \ldots$ & 276.6 & 240 & -98 & 234 & 221 & 96 & 97 & $\mathrm{X}$ & $\mathrm{X}$ \\
\hline $32532 \ldots \ldots \ldots$ & 296.2 & 240 & -212 & 223 & 214 & 85 & 145 & $\mathrm{X}$ & $\mathrm{X}$ \\
\hline $32619 / 20 \ldots \ldots$. & 305.5 & $240 / 80$ & $-493 /-236$ & 233 & 202 & 77 & 132 & 344 & 177 \\
\hline $32717 \ldots \ldots \ldots$ & 324.2 & 190 & -414 & 291 & 141 & $\ldots$ & 111 & 311 & 114 \\
\hline $32797 \ldots \ldots \ldots$ & 338.4 & 210 & -189 & 322 & 170 & 108 & 116 & 411 & $\mathrm{X}$ \\
\hline $32879 \ldots \ldots \ldots$ & 349.5 & 75 & -45 & $\ldots$ & $\ldots$ & $\ldots$ & $\ldots$ & 426 & 162 \\
\hline $32911 \ldots \ldots \ldots$ & 355.1 & 212 & -170 & 338 & 198 & 76 & 95 & 414 & 137 \\
\hline $32938 \ldots \ldots \ldots$ & 360.0 & 180 & -591 & 349 & 174 & 69 & 97 & 425 & 114 \\
\hline $33035 \ldots \ldots \ldots$ & 375.3 & 240 & 85 & 379 & 197 & 116 & 127 & $\mathrm{X}$ & $\mathrm{X}$ \\
\hline 33105/04 ....... & 388.0 & $240 / 70$ & $5 /-211$ & 375 & 211 & 114 & 126 & 482 & 206 \\
\hline $33175 / 76 \ldots \ldots$. & 400.3 & $210 / 90$ & $85 /-292$ & 464 & 195 & 84 & 149 & 442 & 199 \\
\hline $33280 \ldots \ldots \ldots$ & 416.0 & 200 & 621 & 498 & 151 & 110 & 172 & 511 & 197 \\
\hline $33331 / 32 \ldots \ldots \ldots$ & 423.2 & $240 / 75$ & $135 /-186$ & 383 & 202 & 80 & 173 & 444 & 181 \\
\hline $33423 \ldots \ldots \ldots$ & 433.6 & 80 & -40 & $\ldots$ & $\ldots$ & $\ldots$ & $\ldots$ & 338 & 178 \\
\hline $33492 \ldots \ldots \ldots \ldots$ & 440.6 & 80 & 345 & $\ldots$ & $\ldots$ & $\ldots$ & $\ldots$ & 531 & 176 \\
\hline 33497/96 ....... & 441.9 & $240 / 70$ & $3 / 292$ & 385 & 152 & 79 & 137 & 404 & 202 \\
\hline $33519 / 20 \ldots \ldots$. & 445.1 & $240 / 80$ & $-178 / 162$ & 418 & 170 & 98 & 131 & 362 & 215 \\
\hline $33644 \ldots \ldots \ldots$ & 458.3 & 80 & 73 & $\ldots$ & $\ldots$ & $\ldots$ & $\ldots$ & 322 & 211 \\
\hline $33725 \ldots \ldots \ldots$ & 471.4 & 80 & -326 & $\ldots$ & $\ldots$ & $\ldots$ & $\ldots$ & 324 & 143 \\
\hline $33742 / 41 \ldots \ldots$. & 473.8 & $235 / 80$ & $103 / 44$ & 348 & 121 & 73 & 119 & 367 & 241 \\
\hline 33799/800 ..... & 485.0 & $240 / 80$ & $145 / 153$ & 561 & 133 & 85 & 114 & 327 & 178 \\
\hline $33869 \ldots \ldots \ldots$ & 498.7 & 195 & -132 & 343 & 130 & 84 & 147 & 330 & 145 \\
\hline $33937 \ldots \ldots \ldots$ & 510.9 & 240 & 220 & 303 & 113 & 91 & 106 & 321 & $\mathrm{X}$ \\
\hline $33967 / 66 \ldots \ldots$. & 518.7 & $251 / 90$ & $172 /-236$ & 272 & 109 & 112 & 136 & 361 & 144 \\
\hline $34057 / 58 \ldots \ldots \ldots$ & 532.9 & $240 / 80$ & $172 /-183$ & 314 & 118 & 95 & 88 & 266 & 127 \\
\hline $34088 \ldots \ldots \ldots$ & 538.5 & 200 & 347 & 267 & 141 & 75 & 99 & 294 & 120 \\
\hline $34232 \ldots \ldots \ldots$ & 567.5 & 200 & 115 & 245 & 105 & 86 & 92 & 270 & 103 \\
\hline $34441 \ldots \ldots \ldots$ & 593.5 & 150 & -200 & 194 & $\ldots$ & $\ldots$ & $\ldots$ & 199 & 100 \\
\hline $34640 \ldots \ldots \ldots$ & 615.8 & 240 & -283 & 203 & 82 & 40 & 98 & 223 & 111 \\
\hline $34751 \ldots \ldots \ldots$ & 632.7 & 240 & -400 & 197 & 61 & 85 & 72 & 222 & 100 \\
\hline $34871 \ldots \ldots \ldots$ & 650.7 & 163 & -502 & 203 & $\ldots$ & $\ldots$ & 104 & 213 & 89 \\
\hline $35126 \ldots \ldots \ldots$ & 670.7 & 180 & -691 & 190 & 63 & 77 & 114 & 167 & 94 \\
\hline $35308 \ldots \ldots \ldots$ & 686.5 & 220 & -332 & 238 & 74 & 65 & 67 & 184 & 99 \\
\hline $35505 \ldots \ldots \ldots$ & 714.3 & 220 & -453 & 187 & 62 & 54 & 87 & 170 & 68 \\
\hline
\end{tabular}


TABLE $1-$ Continued

\begin{tabular}{|c|c|c|c|c|c|c|c|c|c|}
\hline \multirow[b]{2}{*}{ SWP $^{a}$} & \multirow[b]{2}{*}{$\begin{array}{l}\text { DATE } \\
\text { (days) }\end{array}$} & \multirow[b]{2}{*}{$\begin{array}{c}t_{\text {expo }}^{\mathrm{c}} \\
\text { (minutes) }\end{array}$} & \multirow[b]{2}{*}{$\begin{array}{c}v_{\text {corr }}^{\mathrm{d}} \\
\left(\mathrm{km} \mathrm{s}^{-1}\right)\end{array}$} & \multicolumn{6}{|c|}{$\begin{array}{l}\text { INTEGRATED FLUX } \\
\left(10^{-15} \mathrm{erg} \mathrm{cm}^{-2} \mathrm{~s}^{-1}\right)\end{array}$} \\
\hline & & & & $\begin{array}{l}\mathrm{N} \mathrm{v} \\
1240\end{array}$ & $\begin{array}{l}\text { N IV] } \\
1486\end{array}$ & $\begin{array}{l}\text { He II } \\
1640\end{array}$ & $\begin{array}{l}\mathrm{O} \text { III] } \\
1666\end{array}$ & $\begin{array}{l}\mathrm{N} \text { iII] } \\
1750\end{array}$ & $\begin{array}{l}\text { C III] } \\
1908\end{array}$ \\
\hline $35686 \ldots \ldots$ & 740.3 & 240 & 114 & 176 & 24 & 24 & 73 & 94 & 70 \\
\hline $35822 \ldots \ldots$ & 755.1 & 183 & 33 & 182 & 48 & 33 & 74 & 119 & 106 \\
\hline $35940 \ldots \ldots$ & 771.2 & 260 & -29 & 152 & 55 & 55 & 50 & 112 & 66 \\
\hline $36171 \ldots \ldots$ & 796.2 & 210 & 59 & 135 & 63 & 40 & & 123 & 74 \\
\hline $36258 \ldots \ldots$ & 809.9 & 222 & 136 & 159 & 21 & 45 & 87 & 131 & 49 \\
\hline $36279 \ldots \ldots$ & 813.2 & 260 & 267 & 143 & 68 & 54 & 55 & 129 & 50 \\
\hline $36539 \ldots \ldots$ & 844.0 & 260 & 96 & 134 & 25 & 48 & 52 & 99 & 45 \\
\hline $36578 \ldots \ldots$ & 853.8 & 310 & 0 & 142 & 35 & 51 & & 106 & 54 \\
\hline $36676 \ldots \ldots$ & 870.9 & 260 & 141 & 151 & 28 & 47 & 87 & 101 & 33 \\
\hline $36801 \ldots \ldots$ & 893.9 & 260 & 93 & 129 & 18 & 44 & 55 & 120 & 58 \\
\hline $36968 \ldots \ldots$ & 928.5 & 270 & 89 & 137 & 7 & 28 & 44 & 67 & 43 \\
\hline $37062 \ldots \ldots$ & 936.5 & 230 & -182 & 119 & 1 & 34 & 70 & 102 & 48 \\
\hline $37088 \ldots \ldots$ & 938.8 & 300 & 218 & 115 & 1 & 42 & 54 & 121 & 54 \\
\hline $37236 \ldots \ldots$ & 951.5 & 263 & 873 & 89 & 3 & 36 & 55 & 85 & 26 \\
\hline $37424 \ldots \ldots$ & 970.7 & 230 & -359 & 103 & 5 & 46 & 39 & 108 & 47 \\
\hline $37574 \ldots \ldots$ & 992.6 & 300 & -501 & 122 & 9 & 51 & 55 & 91 & 52 \\
\hline $37798 \ldots \ldots$ & 1021.6 & 300 & -422 & 82 & 42 & 44 & 51 & 64 & 62 \\
\hline $37973 \ldots \ldots$ & 1049.5 & 285 & -314 & 99 & 33 & 69 & 56 & 63 & 39 \\
\hline $38055 \ldots \ldots$ & 1061.1 & 280 & -748 & 92 & -9 & 19 & 60 & 95 & 32 \\
\hline $38172 \ldots \ldots$ & 1081.3 & 285 & -332 & 101 & 4 & 11 & 42 & 69 & 78 \\
\hline $38307 \ldots \ldots$ & 1105.3 & 285 & -424 & 135 & 20 & 5 & 43 & 48 & 19 \\
\hline $38336 \ldots \ldots$ & 1112.0 & 275 & -414 & 115 & -3 & 24 & 44 & 50 & 33 \\
\hline $38536 \ldots \ldots$ & 1137.2 & 285 & 90 & 89 & 7 & 4 & 57 & 51 & 34 \\
\hline $38866 \ldots \ldots$ & 1186.1 & 285 & -152 & 80 & 18 & 13 & 22 & 24 & 34 \\
\hline $39300 \ldots \ldots$ & 1247.0 & 285 & -6 & 50 & 10 & 35 & 55 & 28 & 23 \\
\hline $39757 \ldots \ldots$ & 1317.7 & 265 & -287 & 90 & -11 & 6 & 40 & 35 & 19 \\
\hline $40002 \ldots \ldots$ & 1344.7 & 270 & -550 & 65 & -10 & 53 & 58 & 40 & 10 \\
\hline $40275 \ldots \ldots$ & 1380.5 & 280 & -491 & 43 & 6 & 23 & 28 & 28 & 9 \\
\hline $40858 \ldots \ldots$ & 1449.3 & 280 & -422 & 4 & 2 & 3 & 28 & 19 & $\ldots$ \\
\hline $41179 \ldots \ldots$ & 1490.4 & 310 & -110 & 17 & -5 & 2 & 25 & 16 & 11 \\
\hline $41802 \ldots \ldots$ & 1566.0 & 290 & 198 & 20 & -2 & $\ldots$ & 20 & 10 & 3 \\
\hline $42174 \ldots \ldots$ & 1623.0 & 270 & -372 & 46 & 10 & 32 & 26 & 2 & 15 \\
\hline $43049 \ldots \ldots$ & 1719.6 & 340 & -307 & 37 & -10 & 3 & 22 & 2 & 5 \\
\hline $43393 \ldots \ldots$ & 1755.6 & 340 & -255 & 21 & 1 & 9 & 10 & 11 & -4 \\
\hline $44017 \ldots \ldots$ & 1820.4 & 340 & -678 & 43 & 10 & -3 & 28 & 8 & 7 \\
\hline $44142 \ldots \ldots$ & 1840.3 & 360 & -405 & 17 & 3 & -3 & 24 & 5 & -1 \\
\hline $44442 \ldots \ldots$ & 1882.2 & 270 & -577 & 18 & 19 & 5 & 28 & 9 & 1 \\
\hline
\end{tabular}

${ }^{a}$ SWP image number. On 13 dates two images of different exposure times were taken consecutively to obtain good-quality spectra of all six lines. Data entries corresponding to exposures shorter than 100 minutes are given in italics.

${ }^{\mathrm{b}}$ Observation date, measured in days from the IMB neutrino event, 1987 February 23.316 (Bionta et al. 1987).

${ }^{c}$ Exposure duration in minutes.

${ }^{\mathrm{d}}$ Cross-correlation velocity correction (see $\S 3.3$ ).

e " $\mathrm{X}$ " denotes that the raw spectral data is saturated at this wavelength.

Twenty SWP high-dispersion spectra of SN 1987A were obtained during the UV emission-line phase $(75<t<1800)$ days. These observations and their analysis are discussed below in $\S 4.4$.

Following the rapid decrease of the supernova's UV flux shortward of $2000 \AA$ in the first week of the outburst, the continua of two early-type stars appeared in SWLA spectra of SN 1987A (Kirshner et al. 1987). The identification of the progenitor hinged on close scrutiny of these two previously unknown stars in the LMC located within arcseconds of the supernova's position. Analysis of their spatial separation in SWP low-dispersion spectra taken several weeks after outburst showed that Sk $-69^{\circ} 202$ had disappeared (Gilmozzi et al. 1987; Sonneborn, Altner, \& Kirshner 1987). These same two stars, referred to as star 2 and star 3, after their position in the tabulation of West et al. (1987), have dominated UV flux in SWP large-aperture exposures of SN 1987A since 1987 March. Stars 2 and 3 are offset from SN 1987A by 2".91 (P.A. $\left.=318^{\circ}\right)$ and 1".63 (P.A. $\left.=118^{\circ}\right)$, respec- tively, as measured in recent HST WFPC2 images (M. Romaniello 1996, private communication). These updated positions are within the measurement errors of Walborn et al. (1987).

Several known sources contribute to the observed spectrum in the IUE large-aperture exposures taken at the position of SN 1987A. In addition to the supernova, there is the emission from circumstellar material (the subject of this paper), the continua of stars 2 and 3, and a very faint star located about $9^{\prime \prime}$ from SN 1987A, which enters the end of the SWLA every 6 months when the aperture has the appropriate orientation. These contributions were taken into account in the measurement of emission-line strengths. Stars 2 and 3 are the most important secondary sources for purposes of this paper.

The shape of emission lines and supernova continuum is affected by the detailed structure of the combined stellar spectra. Correcting the observed SWP spectrum requires more than the subtraction of a stellar background spec- 
trum. By virtue of its location near the south ecliptic pole, the LMC is observable 365 days per year and the position angle of the IUE large aperture changes by about 1 degree per day. As the orientation of the SWLA rotates on the sky during the year, the projection of stars 2 and 3 on to the spectrograph dispersion axis also varies. Stars 2 and 3 appear to rotate about the supernova's position (relative to the aperture) and their projection onto the direction of spectral dispersion has a 365 day periodicity. The spatial separation $\left(4^{\prime \prime} .5\right)$ corresponds to $\sim 1200 \mathrm{~km} \mathrm{~s}^{-1}(\sim 7 \AA)$ when the stars are aligned parallel to the dispersion direction. The principal spectral features in the stellar spectra shift in wavelength because star 2 contributes about $70 \%$ of their combined flux below $2000 \AA$.

Accurate centering of the supernova in the SWLA was necessary in order to obtain reliable emission-line light curves. During the time interval spanning the observations, SN 1987A covered over 13 magnitudes of visual brightness. The IUE Fine Error Sensor (FES) was used to acquire the supernova, providing position measurements of its center of light from day $1(V \sim 4.5)$, through visual maximum (day 86, $V \sim 3.0)$, and up to day $\sim 885(V \sim 13.4)$. After that time the supernova was acquired as a blind offset from the nearby star Sk $-69^{\circ} 203,138^{\prime \prime} 5$ almost due N of SN 1987A (P.A. $\left.=2^{\circ} .63\right)$. The coordinates $(\mathrm{B} 1950.0)$ used for the blind offset maneuver are $\alpha=05^{\mathrm{h}} 35^{\mathrm{m}} 48^{\mathrm{s}} \mathrm{s}, \delta=-69^{\circ} 15^{\prime} 39^{\prime \prime} .4$ for Sk $-69^{\circ} 203$ and $\alpha=05^{\mathrm{h}} 35^{\mathrm{m}} 50.0, \delta=-69^{\circ} 17^{\prime} 58^{\prime \prime} .0$ for SN 1987A. The offset maneuver was tested and calibrated between days 700 and 850 when SN 1987A was still bright enough $(12 \lesssim V \lesssim 13)$ to directly acquire it with the FES. The measured maneuver errors on these occasions were \pm 1 FES unit in each axis, or \pm 0 "'.3 total pointing error on the plane of the sky. We therefore believe that there were negligible differences in aperture centering over the 5 years of the observations, which might affect the emission-line spectrophotometry.

\section{DATA ANALYSIS}

The procedures used to measure the emission-line strengths in low-dispersion spectra are described in this section. All line fluxes were measured relative to the net supernova spectrum, that is the observed spectrum with the contribution of stars 2 and 3 subtracted. This procedure, which in principle is the same as used in Paper I, assumes that the relative contributions of stars 2 and 3 to the observed spectrum are constant. This will be true in general provided that the supernova is centered in the SWLA so that both stars are also in the aperture and that the stars themselves are not highly variable in the ultraviolet. This last point is addressed in $\S 4.1$. The principal steps in this process are outlined below.

\subsection{Spatially Resolved Spectral Data}

Our analysis uses the spatially resolved spectra from the extended line-by-line (ELBL) data files produced by the standard IUESIPS processing (Turnrose \& Thompson 1984, as updated by Munoz Piero 1985 for ELBL processing). The ELBL file is a two-dimensional spectral image extracted from the IUE photometrically corrected image along lines of constant wavelength. For the SWP camera it contains about 700 points in the wavelength domain $\left(1150-1980 \AA \AA, \sim 1.2 \AA\right.$ pixel $\left.^{-1}\right)$ and 110 points in the spatial dimension ( 0.68 per linear pixel).
Specialized interactive software was written to correct ELBL pixels corrupted by energetic particle hits and bright spots. For each image the area inside the extraction slit and in the background areas were inspected for bright spots and cosmic-ray effects. When found in the background extraction slit, corrupted pixels were replaced with data values corresponding to the average of immediately adjacent pixels. Extreme care was exercised when correcting bright spots close to the spectrum. Within the extraction slit bright spots were replaced with the average of part of a single row of pixels (parallel to the dispersion) at the same distance above or below the dispersion line.

The spectrum was extracted from the corrected image using a rectangular extraction slit, similar to the standard IUESIPS extraction procedure described by Turnrose \& Thompson (1984). The spatial height of the rectangular spectral extraction slit for the SN 1987A spectrum was 24 lines $\left(16^{\prime \prime}\right)$. For comparison, the IUESIPS standard slit heights are 18 and 30 lines for point source and extended source extractions, respectively. The extraction slit height of 24 lines was chosen to include the wings of the point spread function of stars 2 and 3 at their maximum spatial separation in the spectrograph format, i.e., when their position angle was perpendicular to the dispersion direction. The customized slit height minimizes the background contribution to the extracted spectrum. The standard point source extraction slit is too short, while the standard extended source extraction slit is longer than necessary. Use of a slit-weighted extraction technique such as "optimal" (Kinney, Bohlin, \& Neill 1991) was not used because of the variable, non-point-source nature of these spectra.

The extracted spectra were corrected for two calibration changes. First, a secular shift in the wavelength scale between 1984 and 1988, and second the long-term decline in SWP sensitivity. Starting in 1984 a systematic error developed in the SWP low-dispersion wavelength scale and grew linearly with time. The error resulted from a slow divergence of true dispersion relation from the extrapolation of the mean time-dependent dispersion constants. New dispersion constants were implemented in IUESIPS in 1988 April at GSFC and 1988 September at Vilspa (Thompson 1988). The SWP low-dispersion spectra processed between 1987 February and 1988 September contained an error in the wavelength calibration amounting to $\sim 2.5 \AA$. Thompson (1988) provided an algorithm to fit the time-dependent error in the wavelength scale. This relation was used to correct all SWP low-dispersion spectra of SN 1987A affected by this calibration change.

Long-term changes in the sensitivity of the IUE instrument were compensated for by using the correction scheme of Garhart (1992a). Between 1225 and $2000 \AA$ the SWP low-dispersion sensitivity has decreased linearly since 1979.5 at wavelength-dependent rates of $-0.2 \%$ to $-1.2 \%$ per year. The Garhart sensitivity correction method gives results similar to Bohlin \& Grillmair (1986) for the period in which they overlap. The extracted fluxes were also corrected for the temperature dependence of the SWP camera response $\left(-0.5 \%\right.$ per ${ }^{\circ} \mathrm{C}$, Garhart $\left.1992 \mathrm{~b}\right)$. Finally, the spectral fluxes were placed on the IUE absolute flux scale using the 1980 absolute calibration (Bohlin \& Holm 1980).

\subsection{Reference Spectrum of Stars 2 and 3}

There was a period of a few weeks in 1987 when the SWP large-aperture spectrum of SN 1987A was due almost 
entirely to stars 2 and 3. This occurred between mid-March and early April, before the emission lines appeared. The SWP large-aperture spectra taken during this period were examined to determine which ones could be combined to form a reference spectrum of stars 2 and 3. Criteria included no overexposure, good signal-to-noise ratio at wavelengths shortward of $1500 \AA$ (i.e., $t_{\exp } \gtrsim 160$ minutes), and no apparent signal from SN 1987A, particularly longward of $1800 \AA$. Three spectra were selected: SWP 30592 (day 27.3, $t_{\exp }=$ 300 minutes), SWP 30637 (day 33.1, $t_{\exp }=185$ minutes), and SWP 30743 (day 44.2, $t_{\exp }=225$ minutes). These spectra were reduced in the same manner described in $\S 3.1$. SWP 30637 and 30743 were registered in wavelength with respect to SWP 30592 by cross-correlation. The mean spectrum is the average of the three weighted by the individual exposure times. The resulting SWP spectrum of stars 2 and 3 is shown in Figure 1. This spectrum is similar, though not identical, to that used by Pun et al. (1995) for their study the UV continuum properties of the SN 1987A outburst. They derived their star 2 and 3 UV spectrum by averaging latetime IUE spectra $(1300<t<1500$ days $)$, when the emission lines were extremely weak, in order to recover the UV spectrum of the supernova early in the outburst. In contrast, we use early-time spectra for stars 2 and 3, before the emission lines appear, so that we can follow the decline of the emission lines at late times. Our spectrum of stars 2 and 3 is in good agreement with the FOS spectrum of star 2 (Fig. 1 of Scuderi et al. 1996), given the differences in spectral resolution and calibration between the two instruments. Star 2 contributes $\sim 70 \%$ of the flux in this wavelength region.

\subsection{Background Star Subtraction}

Subtraction of the reference spectrum of stars 2 and 3 from the observed spectrum requires that the two wavelength scales be registered to correct for any offset. Such a wavelength shift could be due to either an offset in the center of light from the center of the SWLA or a shift in the spectral features of stars 2 and 3 in the observed spectrum. In the case of the former, a 1".0 pointing error in the dispersion direction corresponds to a spectral shift of $\sim 300 \mathrm{~km}$

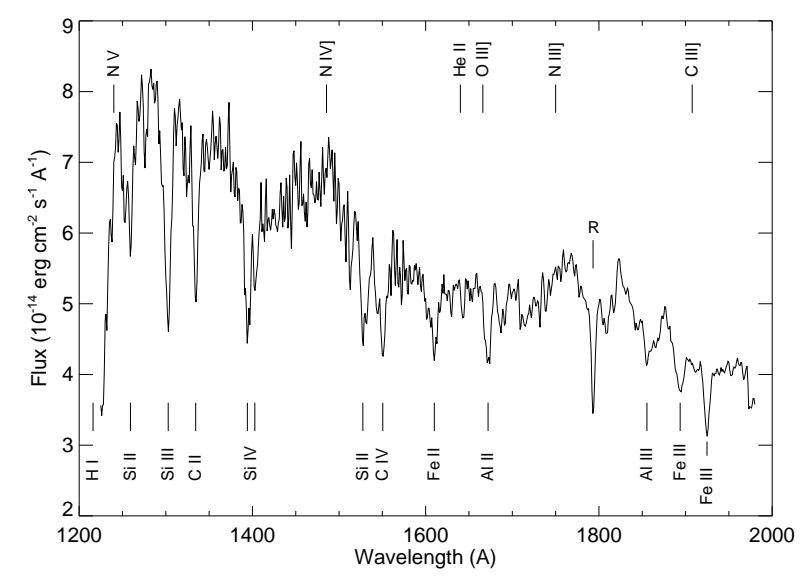

FIG. 1.-Reference SWP spectrum of stars 2 and 3 is the exposureweighted sum of SWP 30592, 30637, and 30743. The principal stellar and interstellar absorption features are identified below the spectrum. The position of the six UV emission lines are marked above the spectrum. A prominent camera reseau is indicated by "R."

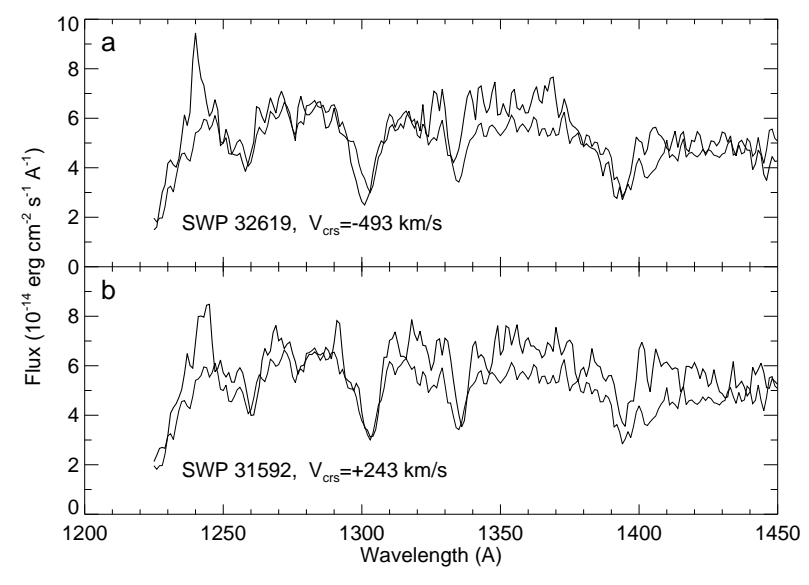

FIG. 2.-Two SWP spectra of SN 1987A (thicker lines) illustrating the effect of the changing orientation of stars 2 and 3 with respect to the dispersion axis. The SWLA position angles for the two spectra differ by $156^{\circ}$. The reference spectrum of stars 2 and 3 (see Fig. 1) is shown by the thinner line.

$\mathrm{s}^{-1}$, or $\sim 1.6 \AA$. The wavelength shift required to properly align stars 2 and 3 with the observed spectrum was computed with an interactive cross-correlation program from the NASA/GSFC IUE Data Analysis Center software library.

Figure 2 shows two SWP spectra with aperture position angles differing by $156^{\circ}$. The shifts in the observed spectra, relative to the reference spectrum of stars 2 and 3 , are evident, as is the difference in the widths in the strong stellar lines. Relative to the reference spectrum, the spectra are shifted by -459 and $+243 \mathrm{~km} \mathrm{~s}^{-1}$, as determined by the cross-correlation analysis. Figure 3 shows the wavelength registration shifts for the SN 1987A SWP data set (Table 1). There is a $\sim 1$ year periodicity in the data, indicating that the position of stars 2 and 3 relative to the spectrographaperture geometry is a major source of this variation. Within about \pm 0 ".5 $\left(\sim \pm 150 \mathrm{~km} \mathrm{~s}^{-1}\right)$ the wavelength registration shifts are dominated by target acquisition and pointing errors. The net spectrum is computed by subtracting stars 2 and 3 from each observed spectrum after shift-

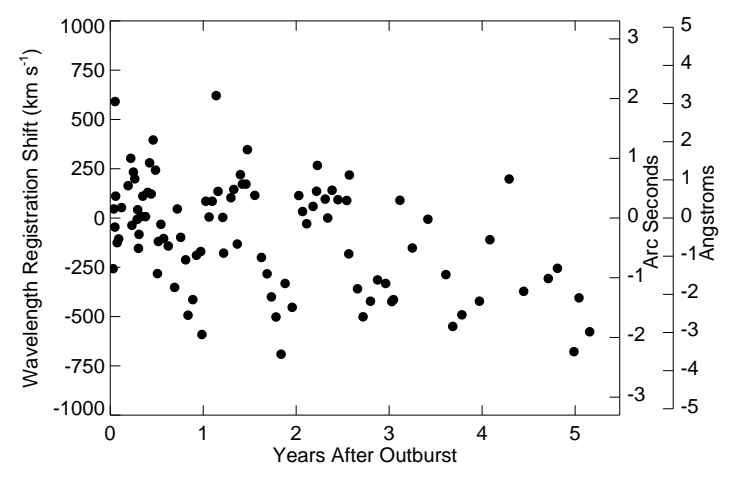

FIG. 3.-Wavelength (velocity) registration shift for the SN 1987A SWP database, as determined by cross-correlation with the star 2 and 3 reference spectrum, shown as a function of time. The right-hand axes show the corresponding spatial and wavelength scales. The scatter at the level of $\pm 125 \mathrm{~km} \mathrm{~s}^{-1}$ is a measure of the accuracy with which the supernova is centered in the aperture. The 1 year cycle of aperture rotation on the sky produces the long-term variation. 
ing the spectrum of stars 2 and 3 by the amount determined by the cross-correlation and interpolating it to the wavelength scale of the observed spectrum.

\section{EMISSION-LINE RESULTS}

\subsection{Fluxes and Light Curves}

The flux in each emission line was measured in the net spectrum where local continuum determination is simplified for most of the emission lines of interest. The subtraction of stars 2 and 3 removes strong stellar and interstellar absorption features, including Si II $\lambda 1260$, Si III $\lambda 1300, C$ II $\lambda 1335$, and $\mathrm{Al}$ III $\lambda 1670$. The wavelengths defining the center and red and blue edges of each line in each spectrum were determined interactively. After subtracting stars 2 and 3 the local continuum was assumed to be flat, except where the SN UV flux is strong. The latter is confined to $\lambda \gtrsim 1700 \AA$ and $t \lesssim 700$ days. The line strength was integrated by a trapezoid algorithm. An example is shown in Figure 4 where the observed and net spectra near the N v line in SWP 36801 (day 894) are shown on the same flux scale. The $\mathrm{N} v$ line is easily distinguished in the net spectrum.

Table 1 contains the fluxes for the $\mathrm{N} v \lambda 1240, \mathrm{~N}$ IV] $\lambda 1486, \mathrm{He}$ II $\lambda 1640, \mathrm{O}$ III] $\lambda 1665, \mathrm{~N}$ III] $\lambda 1750$, and C III] $\lambda 1908$ lines measured in 119 spectra taken on 106 dates. The 1225-1980 ̊ history of SN 1987A between days 85 and 1820 is shown in Figure 5 where the observed net fluxes have been averaged into nine time intervals. The development of the six emission lines is clearly seen. In addition to these emission lines, other features are evident in the net spectra. The supernova continuum longward of $1700 \AA$ is prominent until day $\sim 700$ (see also Pun et al. 1995). There

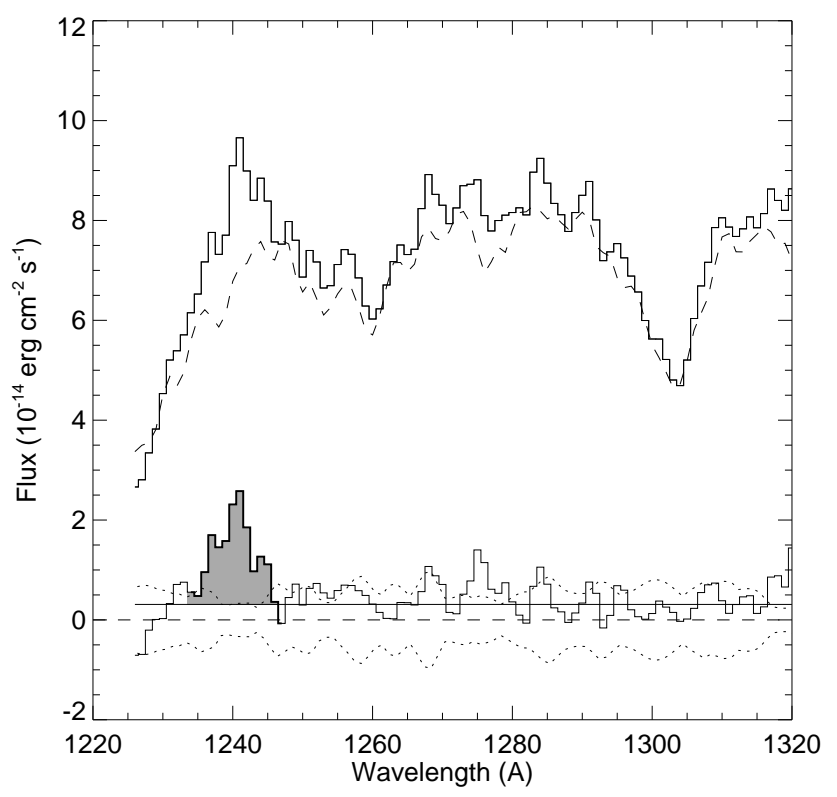

FIG. 4.-Continuum subtraction for $\mathrm{N} \mathrm{v}$ in SWP 36801. The upper solid line is the observed SN 1987A spectrum. The upper dashed line is the reference spectrum of stars 2 and 3 from 1987 March (see Fig. 1). The difference spectrum ( $S N$-stars) is shown by the solid line at the bottom of the figure on the same flux scale. The solid horizontal line is the continuum level established for this $\mathrm{N} v$ flux measurement (shaded area). The dashed horizontal line marks the zero flux level. The $\mathrm{N} \mathrm{v}$ emission feature is more easily distinguished in the difference spectrum. The dotted lines above and below the difference spectrum are the $\pm 1 \sigma$ residuals for the reference spectrum. Cross-correlation techniques were used to register the SN 1987A and star 2 and 3 spectra; a $+93 \mathrm{~km} \mathrm{~s}^{-1}$ correction was applied to the reference spectrum in this example. are additional features that rise and fall in the same time period between 1280 and $1540 \AA$. Of particular interest is the narrow feature at $1400 \AA$, which we tentatively identify with O IV] $\lambda \lambda 1397-1407$. Although we have no identifications for the other features in the spectra, they are believed to be part of the Fe II blanketed continuum of the supernova. The formation of such "pseudo-emission" features in the early spectra of SN 1987A is discussed by Cassatella et al. (1987) and Lucy (1987) and at late times by Fransson (1994) and Li \& McCray (1996). Local maxima occur where line blocking is less important and the photons can escape, i.e., at wavelengths where there are gaps in the list of strong lines. Similar pseudo-emission features occur in classical novae. Hauschildt et al. (1994) modeled and discussed the formation and evolution of these features in Nova Cygni 1992.

The resulting light curves for the $\mathrm{N}$ v, N IV], He II, O III], $\mathrm{N}$ III], and $\mathrm{C}$ III] lines are shown in Figure 6. The light curves show the observed line fluxes, without correction for interstellar extinction (see $\S 5.2$ ). It was sometimes difficult to determine the continuum level at the base of the $\mathrm{C} \mathrm{III]}$ emission line, even after subtracting stars 2 and 3 , because of the UV flux from the supernova debris increased in this region of the spectrum (see Fig. 5). A check of the accuracy of the low-dispersion $\mathrm{C} \mathrm{III]} \mathrm{flux} \mathrm{measurements} \mathrm{was} \mathrm{made} \mathrm{by}$ comparing them with $\mathrm{C}$ III] fluxes from high-resolution observations where continuum determination relative to the narrow emission is much easier. The high-dispersion data for SN 1987A are discussed below (\$ 4.4), and the corresponding $\mathrm{C} \mathrm{III]} \mathrm{fluxes} \mathrm{also} \mathrm{shown} \mathrm{in} \mathrm{Figure} 6 d$. The good agreement between these independent measurements, well within the scatter of the low-resolution data, illustrates the overall accuracy of the continuum determination. The new emission-line light curves are clearly superior to those published previously (Paper I; Sonneborn et al. 1990; Panagia et al. 1991; Sanz Fernández de Córdoba 1993) and may affect results derived from them-for example, Gould (1994, 1995).

The size of the IUE large aperture prompts us to consider the degree to which emission from the outer rings might contribute to the UV light curves, particularly at early times (say, $t<600$ days). If one adopts the geometry suggested by Burrows et al. (1995) the rising branch of the light curve of the southern outer ring (SOR) should extend from about 1 month after explosion to about 3 years later, whereas the northern outer ring (NOR) should have become visible about 2 years after outburst. In this geometry the NOR would not have been entirely visible in the earliest images (Crotts et al. 1989) but may have been "complete" by late 1989 in the ESO images (Wampler et al. 1990). The UV light curves do show a weak secondary maximum around 1000 days, consistent with the epoch when both outer rings would have reached their maximum emission (Panagia et al. 1996, in preparation). Therefore, emission from the NOR would not contribute to the early IUE observations, and emission from the SOR would have contributed less than $\frac{1}{3}$ of its total strength. Present-day HST imaging shows that the NOR, the SOR, and the inner ring have comparable total emission in optical lines. However, the average density in the inner ring is higher than that in the outer rings so the time decay of the inner ring emission would be faster than that of the outer rings. As a consequence, the total emission of the inner ring at the time of its maximum must have been considerably higher than that of the outer rings combined. 


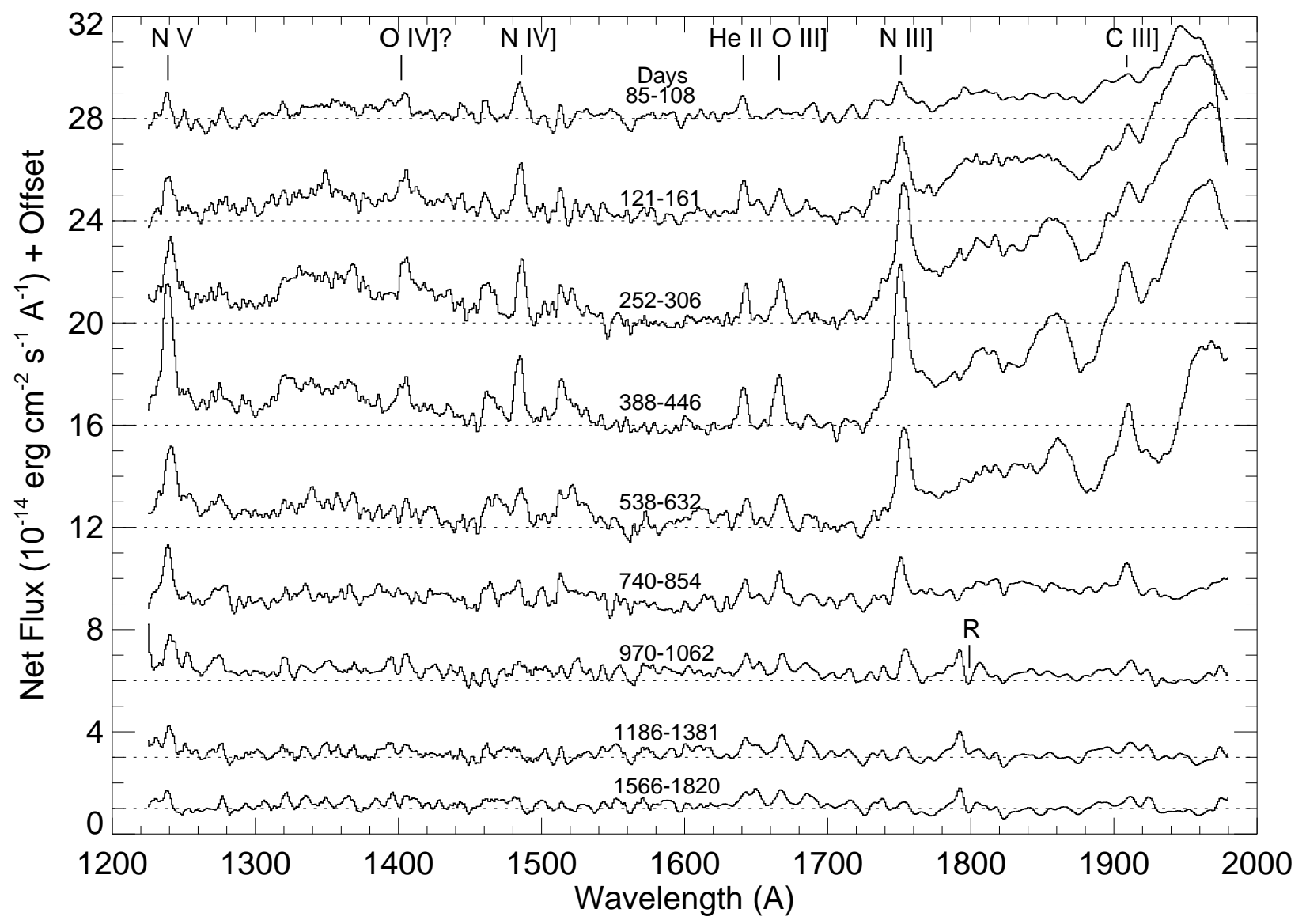

FIG. 5.-Mean SWP spectrum of SN 1987A (minus stars 2 and 3) is shown for nine time intervals spanning the first 1820 days of the outburst. Each mean spectrum is the average of five exposures over the indicated time interval. They are plotted on the same flux scale and are offset for clarity. The dashed horizontal lines mark the zero level for each spectrum. The six emission lines measured are marked at the top of the figure, as is the possible identification of O IV] multiplet UV 0.01 .

In fact, ESO observations around the time of the tentative secondary maximum show that the outer rings contribute less than $10 \%$ of the total [O III] emission (J. Wampler \& L. Wang 1992, private communication). We therefore believe that the contribution of the outer rings to the IUE emission-line light curves is negligible.

Figure 4 shows that the subtraction of stars 2 and 3 is not perfect. Due to fixed-pattern and other small-scale noise, the scatter in the net spectrum near the emission line limits the accuracy of continuum subtraction and hence the significance of short-term fluctuations in the emission-line light curves. The accuracy of the continuum subtraction is quantified by the parameter $\delta F_{c}$, the local continuum residual (eq. [1]), which is the difference between the net spectrum and the adopted local continuum, averaged over a bandpass comparable to the width of the emission line:

$$
\delta F_{c}=\frac{1}{\lambda_{2}-\lambda_{1}} \int_{\lambda_{1}}^{\lambda_{2}}\left[F_{\text {net }}(\lambda)-F_{\text {local }}(\lambda)\right] d \lambda .
$$

The parameter $\delta F_{c}$ was measured near the $\mathrm{N} v$ line because the adjacent continua of stars 2 and 3 have strong stellar and interstellar features, which if not subtracted accurately will add significant and erroneous structure to the net spectrum. Perfect continuum subtraction would yield $\delta F_{c}=0$. $\delta F_{c}$ was computed in a bandpass centered at $1262 \AA$ with a full width of $10 \AA$, the characteristic width of the $\mathrm{N} v$ emission line at zero intensity. Figure 7 shows the $\mathrm{N} v$ light curve (same data as Fig. $6 a$ ) and $\delta F_{c}$. The error bar $\pm \sigma=1.82 \times 10^{-14} \mathrm{erg} \mathrm{cm}^{-2} \mathrm{~s}^{-1}$ corresponds to the measurement uncertainty of individual data points for the six emission lines, indicating that the continuum subtraction procedure works well over the entire 5 year span of the data set.

The accuracy of the continuum subtraction is insensitive to the known variability of the two stars. First, a change in the continuum flux results in a vertical shift of the net spectrum. The continuum in the net spectrum is constant (i.e., horizontal in Fig. 4), except in the presence of the strong SN continuum, so that the emission line is measured relative to this shifted continuum level. Second, it is now well established that only star 3, a Be star, shows any significant variability. Walborn et al. (1993) showed that star 3 has photometric variability ranging from $\pm 0.15 \mathrm{mag}$ in $U$ to $\pm 0.3 \mathrm{mag}$ in $R$ and $I$. Since star 3 contributes only $\sim 30 \%$ of the UV flux, this level of variability is within the measured value of $\sigma$.

The measurement uncertainty of the line fluxes implies that the significant short-term fluctuations in the light curves are astrophysical in origin and are not instrumental effects. The increased scatter in the $\mathrm{N} v$ and $\mathrm{N}$ III] light curves between day 900 and 1100, in the N IV] line before day 400 , and the large values of $\mathrm{N}$ III] and $\mathrm{N} v$ near maximum are actual fluctuations in the emission-line fluxes. We find no evidence that these spectra are affected by cosmic-ray hits or other spurious effects and conclude that 


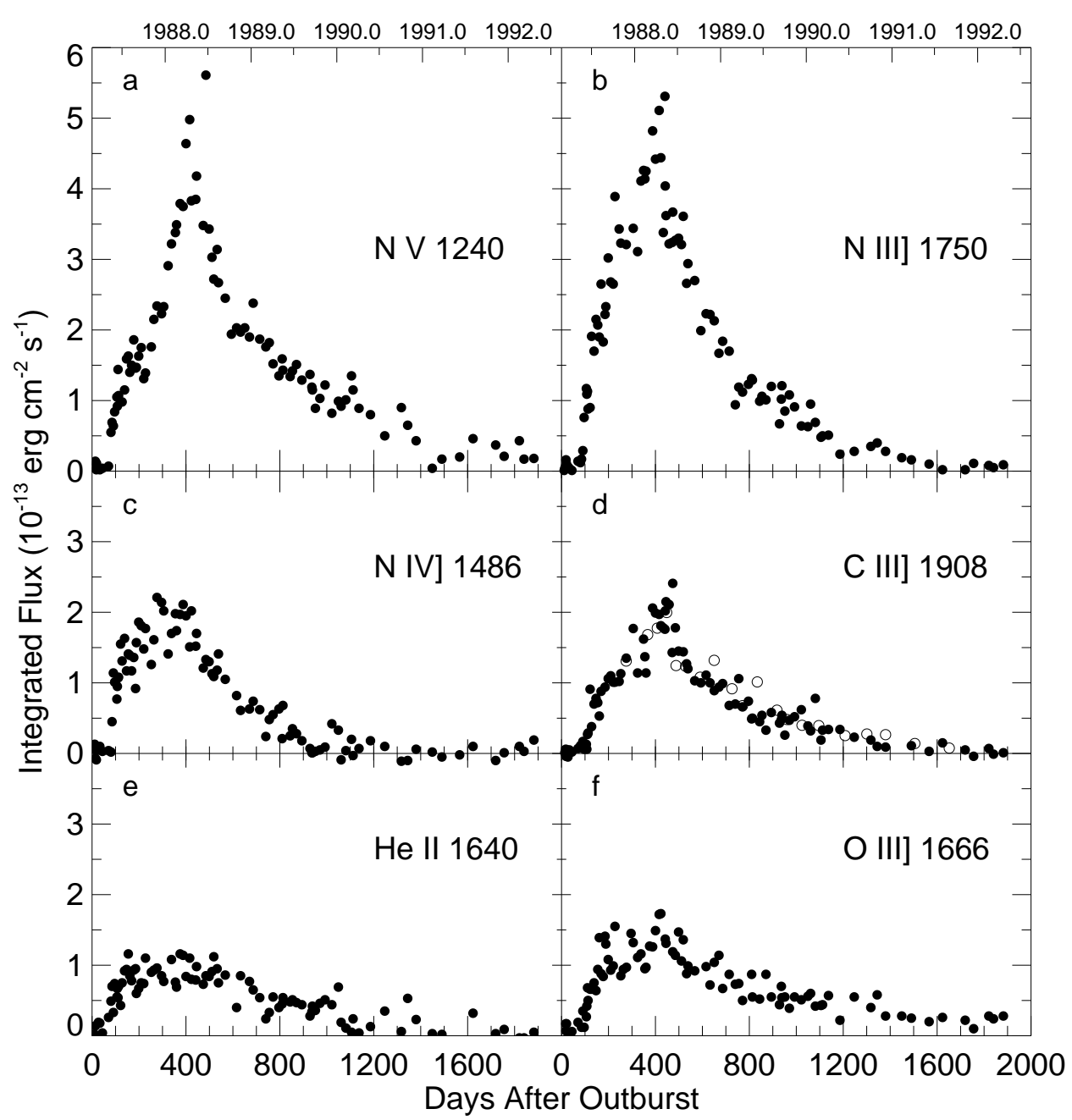

FIG. 6. - Light curves spanning day 11 to 1882 for the six UV emission lines in SN 1987A. The open circles in $(d)$ are the combined C III] $\lambda 1906.68$ and $\lambda 1908.73$ fluxes from the IUE high-dispersion spectra (Table 2).

the fluctuations are real. The largest $\mathrm{N} v$ flux point (day 485) remains suspect, however, as it occurs significantly after maximum brightness and there is no related response in the other lines.

\subsection{Emission-line Maximum}

The times when the lines first appear and when they reach maximum brightness are two important light curve parameters used to estimate the size and inclination of the ring. With a circular ring geometry the emission lines are expected to appear suddenly as the light echo paraboloid makes first contact with the ring (Dwek \& Felten 1992). In general, the highest ionization stage available should be used to measure these parameters.

It is evident from Figure 6 that the six UV lines reach maximum at approximately the same time. However, only the $\mathrm{N} \mathrm{V}$ and $\mathrm{N} \mathrm{III]}$ lines have large fluxes and well-defined maxima necessary for a more quantitative analysis. The actual times of maxima are $t_{\max }^{\mathrm{N} I I I}=399 \pm 15$ and $t_{\max }^{\mathrm{N} \mathrm{V}}=429$ \pm 19 days ( $418 \pm 12$ days if the high data point at day 485 is excluded), as determined by high-order polynomial fits to the light curves. Comparison of the $\mathrm{N} \mathrm{v}$ and $\mathrm{N} \mathrm{III]} \mathrm{in} \mathrm{Figure}$ 8 shows that while their post-maximum decline is very similar, the light curves before maximum have significant differences. Figure 8 shows a large deficiency of $\mathrm{N} v$ relative to $\mathrm{N} \mathrm{III]}$ between 150 and 400 days and that $\mathrm{N} v$ peaks about 3 weeks after $\mathrm{N}$ iII].

The $N$ v $\lambda 1240$ emission from the far side of the ring may be delayed by $1-2$ weeks by resonance scattering in the ring and the bipolar nebula, as discussed by LF96. However, to obtain an even longer delay, and thus later $t_{\max }^{\mathrm{NV}}$, like the one observed, additional resonance scattering is needed. The most likely source is in the $\mathrm{H}$ II region interior to ring discussed by Chevalier \& Dwarkadas (1995). Preliminary calculations by us show that there may be a sufficient optical depth in $N$ v $\lambda 1240$ through this region along the line of sight to the far side of the ring. The large deficiency of $\mathrm{N} v$ relative to $\mathrm{N} \mathrm{III]}$ is therefore probably due to resonant scattering of $\mathrm{N} \mathrm{v}$ photons by $\mathrm{N}^{+4}$ ions in and outside the ring. This biases the $\mathrm{N} v$ flux maximum and complicates its interpretation by introducing effects other than light travel time and local recombination. In view of the uncertainties in interpreting the $\mathrm{N} v$ light curve, we believe $t_{\max }^{\mathrm{N} \text { III }}$ provides the best measure of the time of maximum, $t_{\max }$, and we use this in our subsequent analysis.

\subsection{Emission-line Turn-on}

Figure 9 shows the $\mathrm{N}$ III], N IV], and $\mathrm{N} v$ fluxes up to day 120. All three lines appear very abruptly, as predicted. There is also an ionization dependence in the turn-on times. 


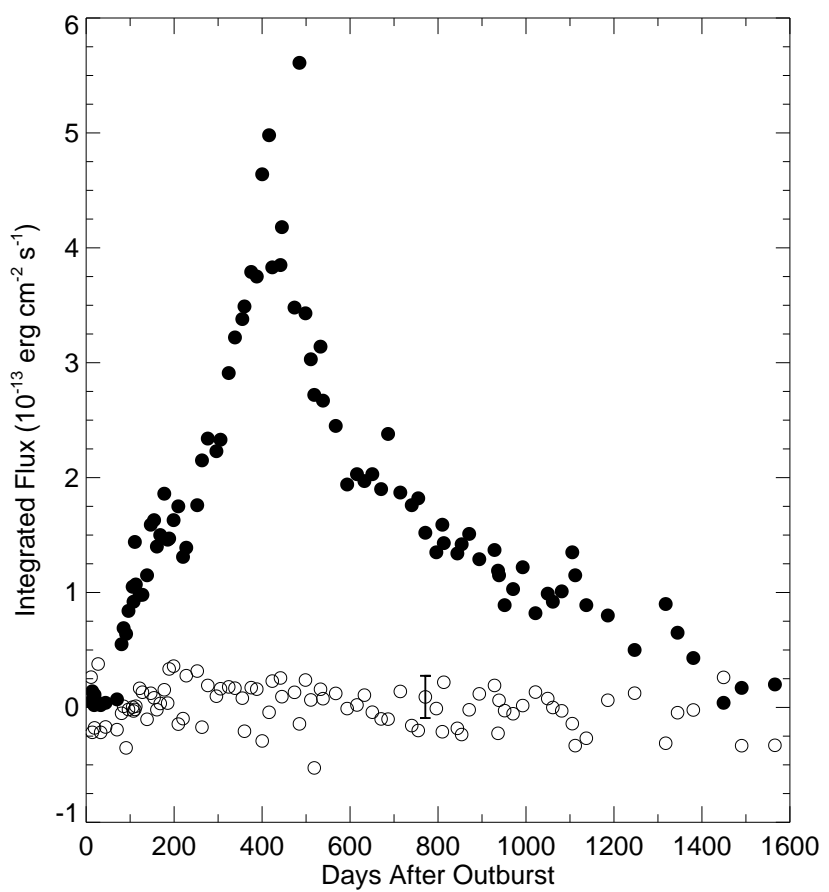

FIG. 7. - N v $\lambda 1240$ light curve ( filled circles) and continuum residual, $\delta F_{c}$ (eq. [1]), near the line are shown as a function of time. $\delta F_{c}$ is a measure of the accuracy and consistency of the continuum placement used to compute the flux in the line (see Fig. 4). The results shown for $\mathrm{N} \mathrm{v}$ are representative of the six lines measured. The error bar $( \pm 1 \sigma=$ $1.82 \times 10^{-14} \mathrm{erg} \mathrm{cm}^{-2} \mathrm{~s}^{-1}$ ) is the measurement uncertainty for individual data points.

$\mathrm{N} v$ appeared first, after day 70.2 and before day 80.6. N Iv] appeared next, between 80.6 and 85.2 days. $\mathrm{N}$ III] appeared last, between 85.2 and 90.9 days. While it is possible that $\mathrm{N}$ III] was present at a very weak level between day 70 and

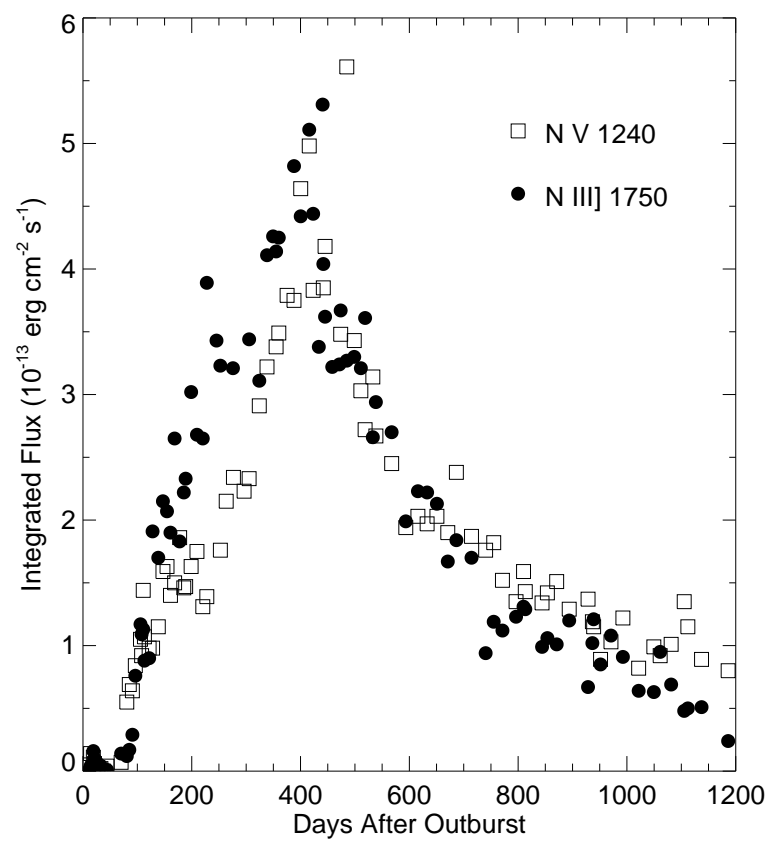

FIG. 8.-Comparison of the $\mathrm{N}$ III] and $\mathrm{N} v$ light curves near maximum. The large deficiency of $\mathrm{N} v$ relative to $\mathrm{N}$ III] before maximum is believed to be the result of resonant scattering by $\mathrm{N}^{+4}$ ions in the highly ionized gas close to the ring along the line of sight to the supernova.

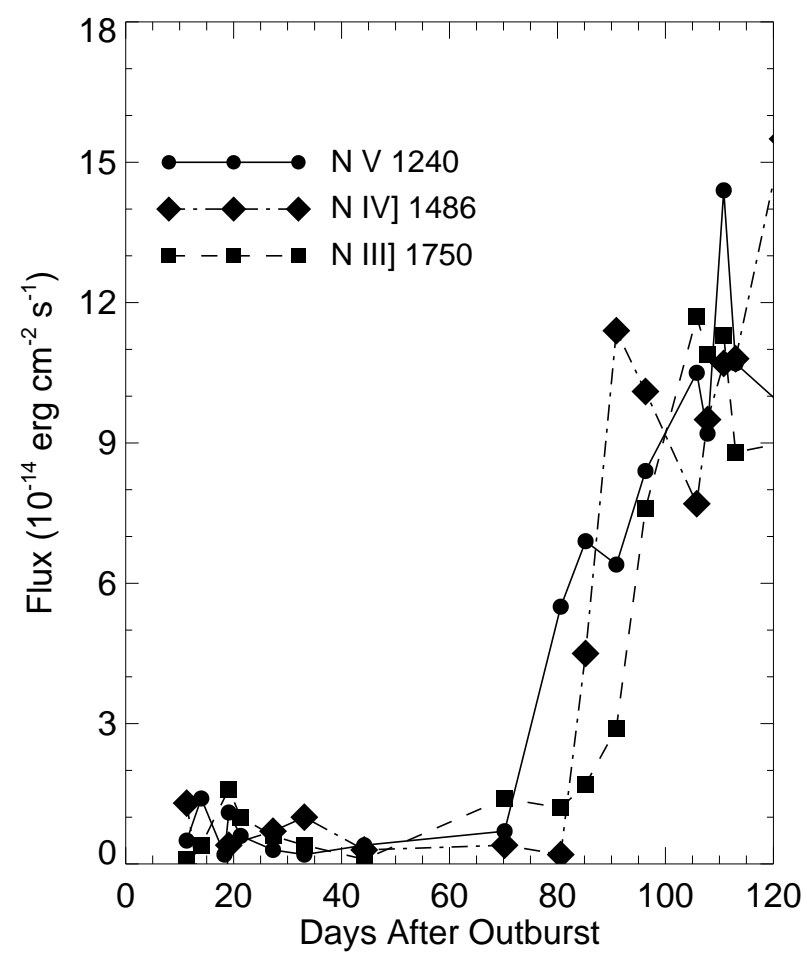

FIG. 9.-Comparison of the three nitrogen emission-line light curves before day 120. The sudden onset of the lines also indicates a progression from higher to lower ionization potential.

85 , the measurements are consistent with zero flux based on the scatter before day 60 . Since the semiforbidden lines are not affected by scattering, $\mathrm{N} \mathrm{IV}]$, the next highest ionization state after $\mathrm{N} \mathrm{v}$, should closely track the inside edge of the ring. However, as discussed below (see also Fig. 6 in LF96), the $\mathrm{N} v$ light curve is significantly affected by resonance scatterings that introduce large systematic errors. Consequently, we believe $\mathrm{N} v$ is also inappropriate for measuring $t_{\text {rise }}$. We take the sharp rise in the N IV] flux as the turn-on time and find $t_{\text {rise }}=83 \pm 4$ days (estimated error). The uncertainty in the turn-on time is set by the spacing of the observations, and consequently $t_{\text {rise }}$ may not be resolved in the IUE data.

On the near side of the ring resonance scattering of $\mathrm{N} \mathrm{v}$ by $\mathrm{N}^{+4}$ ions in the ring and any other intervening highly ionized circumstellar gas should delay the turn on of this line compared to that of other nitrogen lines. Instead, the observations show an earlier turn on of $\mathrm{N} \mathrm{v}$. The most likely explanation to this apparent inconsistency is the ionization stratification of the ionized gas. The effect of stratification on the turn on of $\mathrm{N} \mathrm{V}$ should be larger if there is an $\mathrm{H}$ II region interior to the ring from which there also may be weak N v emission. Another possible source of early N v emission is the bipolar nebula close to the ring, but away from its equatorial plane where the nebula may not be ionization bounded. This part of the nebula may have a lower density than the ring causing it to glow early in $\mathrm{N} \mathrm{v}$, but not in other UV lines due to long recombination timescales.

\subsection{High-Dispersion Spectra}

The first SWP high-dispersion spectrum of SN 1987A during the narrow emission-line phase was taken on 1987 Nov. 25 (day 275) and used in Paper I. Nineteen additional 
TABLE 2

High-DisPERSION C III] AND N III] EMISSION-LINE FLUXES

\begin{tabular}{|c|c|c|c|c|c|c|}
\hline SWP & Date $^{a}$ & $t_{\text {expo }}^{\mathrm{b}}$ & $F_{1907}^{\mathrm{c}}$ & $F_{1909}^{\mathrm{c}}$ & $F_{1749}^{c}$ & $F_{1752}^{\mathrm{c}}$ \\
\hline 32394. & 275.544 & 450 & 6.277 & 7.085 & 15.84 & 12.63 \\
\hline $32983 .$. & 367.110 & 540 & 7.513 & 9.318 & 19.64 & 12.72 \\
\hline $33215 \ldots \ldots$ & 408.167 & 880 & 7.924 & 9.832 & 23.76 & 15.12 \\
\hline $33536 \ldots \ldots$ & 448.026 & 900 & 11.973 & 7.996 & 22.17 & 14.53 \\
\hline $33810 \ldots$ & 487.901 & 850 & 7.139 & 5.311 & 18.96 & 9.84 \\
\hline $34024 \ldots$ & 528.874 & 952 & 6.790 & 5.560 & 16.25 & 12.52 \\
\hline 34420 .. & 590.622 & 760 & 6.229 & 4.578 & 14.07 & 8.43 \\
\hline $34870 \ldots \ldots$ & 650.340 & 612 & 8.094 & 5.099 & 10.57 & 5.72 \\
\hline $35577 .$. & 726.232 & 925 & 5.634 & 3.530 & 12.69 & 6.15 \\
\hline $35929 \ldots \ldots$ & 771.117 & 630 & 4.303 & 3.468 & & 6.90 \\
\hline $36399 \ldots \ldots$ & 834.004 & 880 & 6.821 & 3.311 & 9.96 & 7.59 \\
\hline $36891 \ldots \ldots$ & 917.794 & 945 & 3.234 & 2.897 & 5.48 & 3.10 \\
\hline $37297 \ldots \ldots$ & 960.7 & 975 & 3.236 & 1.567 & 8.05 & 3.44 \\
\hline $37805 \ldots$ & 1024.533 & 970 & 2.512 & 1.481 & 17.34 & 3.57 \\
\hline $38230 \ldots \ldots$ & 1096.065 & 610 & 2.576 & 1.372 & 5.42 & 2.69 \\
\hline $39093 \ldots \ldots$ & 1208.039 & 965 & 1.623 & 0.907 & 6.66 & 5.04 \\
\hline $39644 \ldots \ldots$. & 1300.799 & 930 & 1.803 & 0.954 & 5.98 & 3.29 \\
\hline $40268 \ldots \ldots$ & 1380.515 & 1010 & 1.589 & 1.071 & & 2.81 \\
\hline $41316 \ldots \ldots$ & 1505.059 & 625 & 0.865 & 0.546 & 3.27 & 2.35 \\
\hline $42370 \ldots \ldots$ & 1651.787 & 960 & 0.508 & 0.253 & 3.81 & 1.73 \\
\hline
\end{tabular}

${ }^{a}$ Exposure midpoint measured in days since 1987 February 23.316 UT.

${ }^{\mathrm{b}}$ Exposure time duration in minutes.

${ }^{c}$ Integrated line flux in units of $1 \times 10^{-14} \mathrm{erg} \mathrm{cm}^{-2} \mathrm{~s}^{-1}$.

high-dispersion SWP spectra were obtained between then and day 1652. The observations are summarized in Table 2. Using standard IUESIPS high-dispersion processing, extracted spectra were calibrated using the flux calibration of Cassatella et al. (1994), which provides an accuracy of

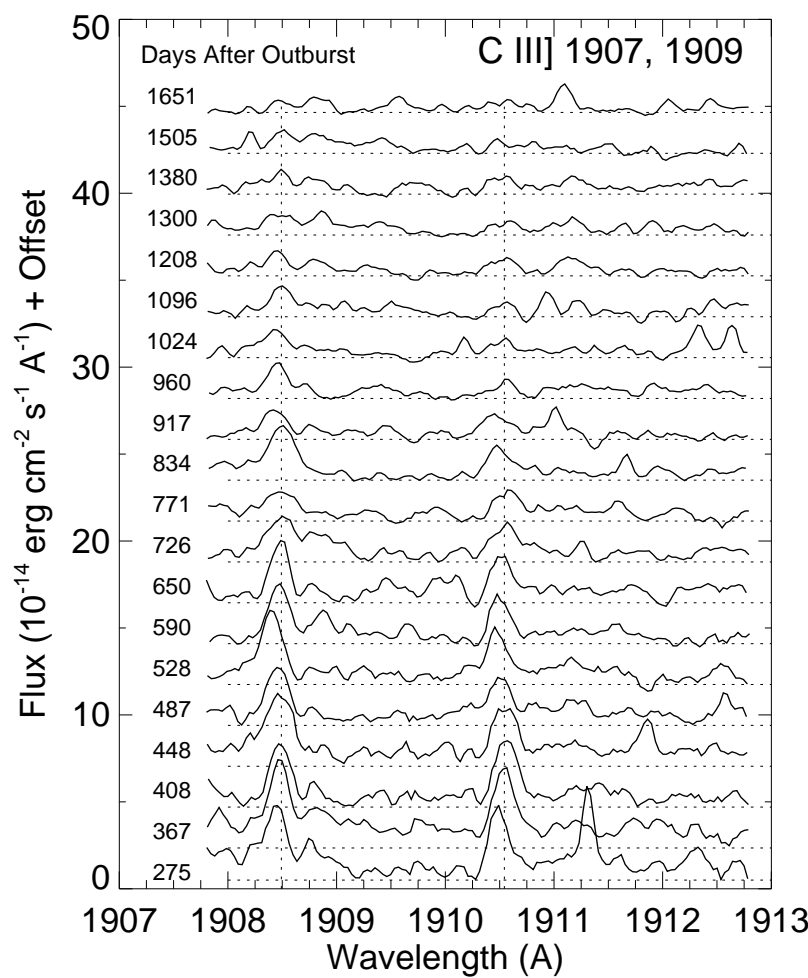

FIG. 10.-Line profiles of $\mathrm{C}$ III] multiplet UV 0.01 detected in IUE high-dispersion spectra of SN 1987A between days 275 and 1652. The two vertical dashed lines mark the positions of the $\lambda 1906.68$ and $\lambda 1908.73$ components at a heliocentric velocity of $+285 \mathrm{~km} \mathrm{~s}^{-1}$.

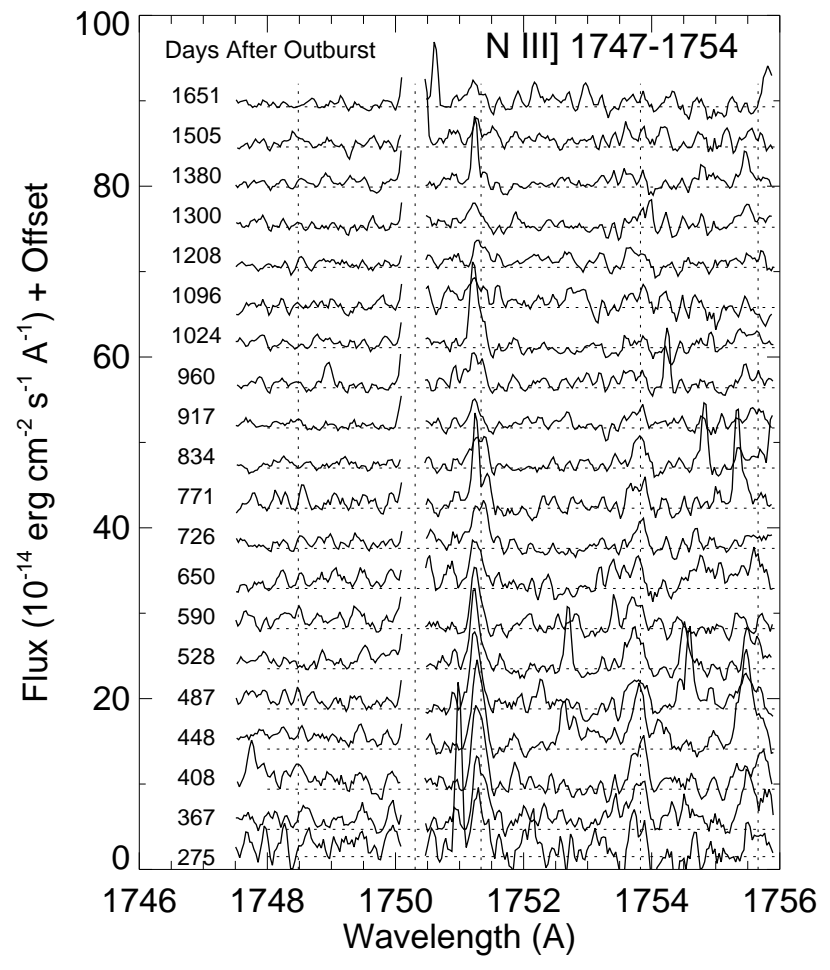

FIG. 11.-Line profiles of $\mathrm{N}$ III] multiplet UV 0.01 detected in IUE high-dispersion spectra between days 275 and 1652 . The five vertical dashed lines mark the positions of the multiplet components at a heliocentric velocity of $+285 \mathrm{~km} \mathrm{~s}^{-1}$.

about $4 \%$ with respect to low-resolution spectra longward of $\sim 1400 \AA$ and increasing by no more than a factor of 2 at shorter wavelengths. All the lines seen in low-resolution were also detected in high-dispersion spectra taken near peak brightness. However, only the $\mathrm{N} \mathrm{III]} \mathrm{and} \mathrm{C} \mathrm{III]} \mathrm{lines}$ were detected over a sufficiently long time base and with sufficient signal-to-noise to be able to discuss their evolution and compare them with the low-dispersion data.

Table 2 also contains the fluxes for C III] $\lambda \lambda 1906.68$, 1908.73 and $\mathrm{N} \mathrm{III]} \lambda \lambda 1749.68,1752.16$. The other members of the $\mathrm{N} \mathrm{III]} \mathrm{multiplet} \mathrm{were} \mathrm{either} \mathrm{too} \mathrm{weak} \mathrm{or,} \mathrm{in} \mathrm{the} \mathrm{case} \mathrm{of}$ $\lambda 1748.61$, badly affected by a camera reseau. Figures 10 and 11 show the spectra for these lines. The line widths are consistent with instrumental broadening (FWHM $\sim 0.18 \AA$ at $\mathrm{N}$ III] and $\sim 0.20 \AA$ at $\mathrm{C}$ III]) for the time period where line widths are reliably measured (up to day $\sim 1000$ for C III]).

The relative strengths of the $\mathrm{C}$ III] and $\mathrm{N}$ III] multiplet components are well-known diagnostics of electron density (Nussbaumer \& Schild 1979; Nussbaumer \& Storey 1979; Berrington 1985). In Paper I we showed that $n_{e}=1-3 \times 10^{4}$ $\mathrm{cm}^{-3}$ at the time of the first high-dispersion spectrum in Table 2 (day 275). The larger number of spectra now available makes it possible to examine the line ratios for evidence of density variations. Figure 12 shows the $\mathrm{C}$ III] $\lambda 1907 / \lambda 1909$ and $\mathrm{N}$ III] $\lambda 1750 / \lambda 1752$ line ratios before day 1000. Calculated $I_{1907} / I_{1909}$ intensity ratio as a function of electron density are shown in Figure 13 for three temperatures using a six-level $\mathrm{C}$ III atom and recent atomic data. The $C$ III] line ratio of $0.8 \pm 0.2$ up to day 408 , implies $n_{e}=4.7 \pm 2.0 \times 10^{4} \mathrm{~cm}^{-3}$, assuming $T_{e} \sim 50,000 \mathrm{~K}$. After $\sim 600$ days, $I_{1907} / I_{1909} \sim 1.5 \pm 0.4$. The average electron 


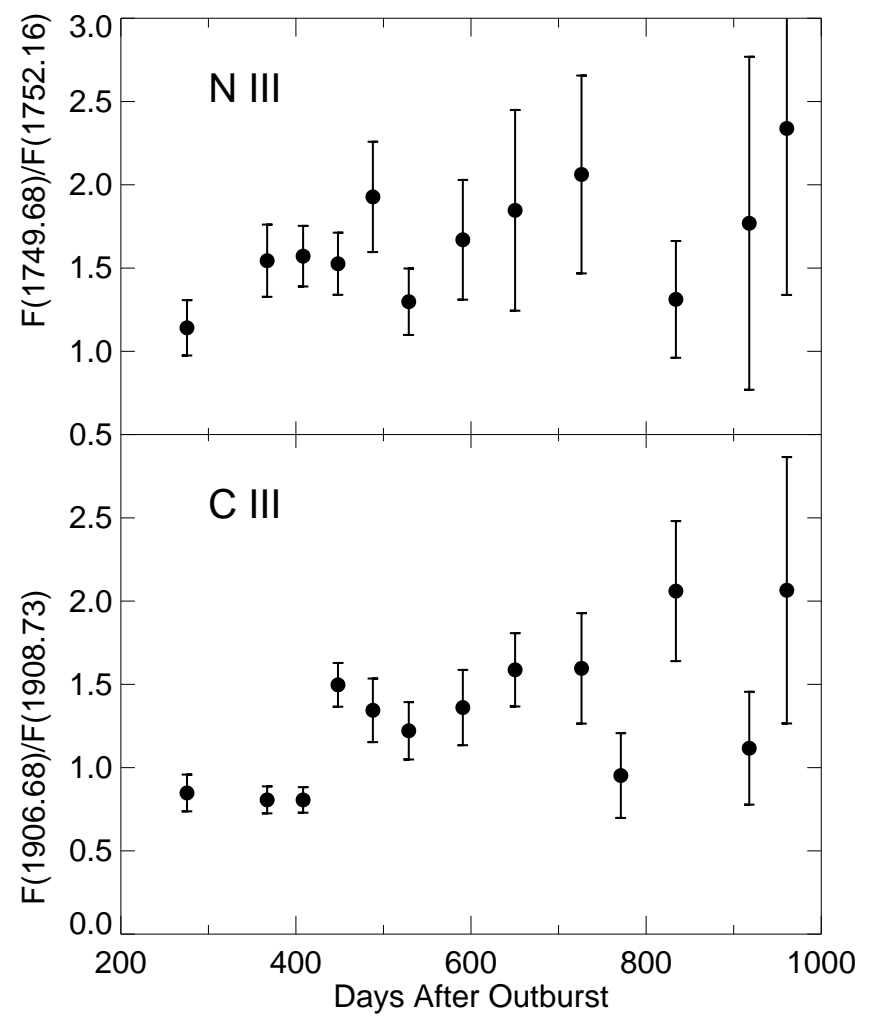

FIG. 12.-Evolution of the N III] $\lambda 1749 / \lambda 1752$ and C III] $\lambda 1907 / \lambda 1909$ line ratios between days 275 and 1000 .

density for the $\mathrm{C} \mathrm{III]} \mathrm{emitting} \mathrm{gas} \mathrm{decreases} \mathrm{by} \mathrm{a} \mathrm{factor} \mathrm{of}$ $\sim 3$ or more from $\sim 5 \times 10^{4} \mathrm{~cm}^{-3}$ before $\sim 400$ days to $\lesssim 1-2 \times 10^{4} \mathrm{~cm}^{-3}$ thereafter, depending on $T_{e}$. Near $\sim 600$ days $T_{e} \sim 30,000 \mathrm{~K}$ may be more appropriate, favoring the

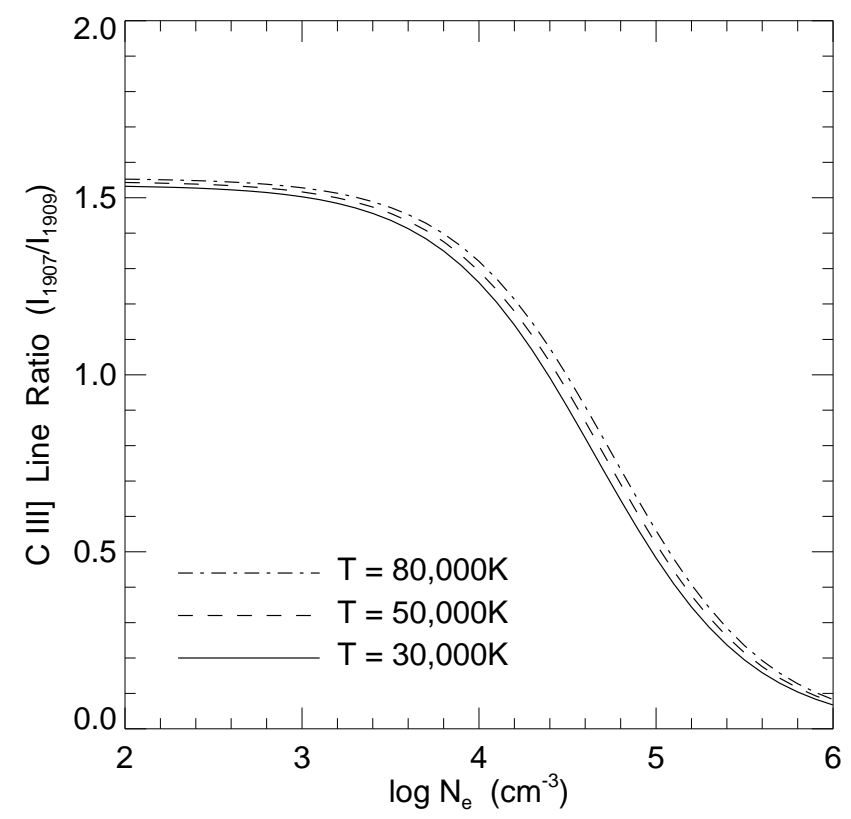

FIG. 13.-Calculated C III] line ratios $\left(I_{1907} / I_{1909}\right)$ as a function of $n_{e}$ for a six-level C III atom are shown for electron temperatures of 30,000 K, $50,000 \mathrm{~K}$, and $80,000 \mathrm{~K}$. The atomic data are from several sources: $A$-values for C III from Nussbaumer \& Storey (1978), Allard et al. (1990), and Fleming et al. (1994), energy levels are those of Mendoza \& Zeippen (1983) and Aggarwal et al. (1986), and collision strengths from Seaton (1987) and Keenan et al. (1992). lower estimate of $n_{e}$. The increasing uncertainty in the later data points makes the line ratios after $\sim 800$ days unreliable for density analysis.

\section{DISCUSSION}

The absolute dimensions of the emitting region can be derived from the shape of the UV light curves with some basic assumptions about its geometry, mainly a circular thin ring. For this purpose we summarize the main features of the recombination/cooling-light echo scenario. Hydrodynamic models of the breakout of the supernova shock wave through the photosphere of the progenitor show that a strong pulse of soft X-rays is emitted with a duration of a few hours (Shigeyama, Nomoto, \& Hashimoto 1988; Woosley 1988; Shigeyama \& Nomoto 1990; Blinnikov \& Nadyozhin 1991; Ensman \& Burrows 1992). The photospheric temperature, and also the radiation temperature, decrease from $\sim 10^{6} \mathrm{~K}$ to $\sim 2 \times 10^{4} \mathrm{~K}$ in about 12 hours. The ionizing flux from the supernova is therefore exclusively emitted during the first day, and in contrast to the interstellar visual light echoes, we can consider the emission from the supernova as instantaneous. The soft $\mathrm{X}$-rays ionize the circumstellar gas on a timescale of hours, leading to highly ionized species like the observed $\mathrm{N} \mathrm{v}$, or even higher (LF91; LF96). At the same time the radiation heats the gas to temperatures exceeding $\sim 10^{5} \mathrm{~K}$. The subsequent evolution of the gas, occurring on a timescale of weeks to years, is determined by the coupled cooling and recombination of the plasma.

\subsection{Geometry}

The recombination and cooling times are set by the temperature and density, $t_{\mathrm{rec}} \sim 1 /\left(\alpha_{\mathrm{rec}} n_{e}\right)$, so gas at low density recombines and cools on a longer timescale. The finite velocity of light implies that only a region bounded by the light echo paraboloid, given by $r=c t /(1-\cos \theta)$, can be observed from the Earth at time $t$. Here $r$ is the distance from the supernova, $t$ is the time of the observation, and $\theta$ is the angle between the line of sight and the direction to the emitting gas as seen from the supernova. In the case of a spherical shell, with radius $R_{s}$, the whole structure is seen after a time $t_{\max }=2 R_{s} / c$, while for a circular ring $t_{\max }=$ $(1+\sin i) R / c$, where $R$ is the ring's radius and $i$ is the inclination of the plane of the ring with respect to the line of sight ( $i=0^{\circ}$ is face-on). For ions that recombine and cool slowly, the observed flux increases nearly linearly up to $t_{\max }$. If the density is high enough recombination occurs rapidly and the light curves of different ions reach maximum simultaneously followed by decay on the individual recombination timescales. In the slow recombination case the light curves will remain at a roughly constant level after maximum.

LF91 showed that for a spherical shell the gas was marginally optically thin in the continuum. In the case of a ring geometry, however, the gas must have significant optical depth in the EUV continuum because of the larger column density needed to produce the same emission (LF96). If the ionization of the gas were radially constant (as is probably not the case), the light curves of medium ionization ions formed by recombination, like $\mathrm{N}$ III, would be delayed with respect to those formed by photoionization at the time of the outburst, like $\mathrm{N}$ v. However, from the outset the ring will be optically thick to ionizing photons, resulting in a 
radial ionization gradient with several ionization zones (Lundqvist 1992). Medium ionization ions like N III and $\mathrm{N}$ IV are created with high abundances outside the $\mathrm{N} \mathrm{v}-$ $\mathrm{N}$ vi zone. Models using a ring geometry are discussed in detail by LF96 and are consistent with the observed light curves. The delayed turn-on of $\mathrm{N} \mathrm{IV]}$ and $\mathrm{N} \mathrm{III]} \mathrm{(Fig.} \mathrm{8)}$ relative to $\mathrm{N} v$ supports this model.

The HST images clearly resolve the ringlike shape of the emitting gas in line and continuum radiation (Jakobsen et al. 1991, 1994; Plait et al. 1995; Burrows et al. 1995). While the extent of the region perpendicular to the ring plane remains to be determined by modeling of the lines, there are additional indications from the IUE observations for a thin ring geometry. The most direct evidence for this comes from the abrupt rise of the emission-line fluxes. As discussed in $\S 4.1$, we adopt the $\mathrm{N}$ IV] turn-on time $\left(t_{\text {rise }}=83 \pm 4\right.$ days $)$ as the initial rise time. In $\S 4.1$ we also determined $t_{\max }=$ $399 \pm 15$ days from the $\mathrm{N}$ III] line. The delay from an inclined circular ring is given by $t_{\text {rise }}=(1-\sin i) R / c$. We therefore have $R=c\left(t_{\max }+t_{\text {rise }}\right) / 2=(6.24 \pm 0.20) \times 10^{17}$ $\mathrm{cm}$, and $\sin i=c\left(t_{\max }-t_{\mathrm{rise}}\right) / 2 R=0.656 \pm 0.039$, or $i=$ $41.0 \pm 3.9$. The inclination has also been measured from the HST FOC images, assuming a circular ring. Jakobsen et al. (1991) found $i=43^{\circ} \pm 3^{\circ}$, and Plait et al. (1995) found $i=44.0 \pm 1.0$. The agreement between these values shows that the ring is nearly circular (see also Gould 1994; Lundqvist 1994; Crotts et al. 1995).

Our value of $R$ has direct bearing on the estimated distance to SN 1987A using the method devised by Panagia et al. (1991) and refined by Gould (1994, 1995). Gould finds $t_{\text {rise }}=75.0 \pm 2.6$ days and $t_{\max }=390 \pm 1.8$ days, which combined with the angular size of the ring in [O III] at $t>1200$ days from Plait et al. (1995) gives a distance $d_{\mathrm{SN}}<$ $46.77 \pm 0.76 \mathrm{kpc}$. Our slightly larger values of $t_{\text {rise }}$ and $t_{\max }$ and more realistic errors translate directly into a larger value and uncertainty for the distance, $d_{\mathrm{SN}}=48.6 \pm 2.2 \mathrm{kpc}$. A more complete statistical treatment of the light curves and the time of maximum and related systematic errors affecting the distance to the supernova is beyond the scope of this paper but is discussed by Lundqvist et al. (1997, in preparation). We emphasize that systematic errors may be significantly larger than statistical errors for several reasons. For example, the method assumes perfect circularity of the ring. Although this may be a reasonable approximation for the overall geometry of the ring, there may be local variations of the radius around the ring. In particular, a small inward kink or near absence of gas on the far side of the ring would give a smaller $t_{\max }$ than for a circular ring, thus underestimating $d_{\mathrm{SN}}$. Second, the structure of the [O III] ring at $t>1200$ days was probably different from the structure of the gas emitting the UV lines at $t \lesssim 400$ days. Indeed, LF96 found that the ionization structure of the ring changes dramatically in only a few hundred days. The UV lines are somewhat more sensitive to temperature and somewhat more peaked toward the inner edge of the ring than forbidden line emission (e.g., [O III]). The analyses of Panagia et al. and Gould may be affected by a systematic error arising from the assumption that the optical forbidden lines and UV lines come from the same gas (but not observed at the same epoch). Depending on the radial distribution of the density in the ring, the [O III] structure could either have a smaller, or larger, mean radius than the early UV line emission. We also point out that their analyses used UV line fluxes from two independent reductions (labeled GSFC and Vilspa) shown by Panagia et al. (1991) of the same set of spectra, thus the number of independent data points is too large by a factor of 2 .

\subsection{Abundances}

The increased number of observations compared to Paper I, reduced measurement and systematic errors, and improved signal-to-noise ratios make it important to repeat the abundance analysis. In order to convert the ionic ratios obtained from the line ratios to elemental abundances, assumptions about these relations have to be made. More specifically, in Paper I it was assumed that N/C = N III/C III and $\mathrm{N} / \mathrm{O}=(\mathrm{N}$ III $+\mathrm{N}$ Iv $) / \mathrm{O}$ III. The calculations by LF91 and LF96 show that, because of a balance of recombinations and collisional ionizations, this is a good approximation until the decay of the lines begin. After this epoch the gas temperature is too low for collisional ionization to be important for $\mathrm{C} \mathrm{III,} \mathrm{N} \mathrm{III,} \mathrm{and} \mathrm{O}$ III, and the lines decay on their respective recombination timescales. These are quite different for the ions involved, and consequently the relations above break down. Before $\sim 200$ days the lines were weak and the fluxes therefore uncertain. Consequently, in this analysis we restrict ourselves to $200<t<500$ days. The collision strengths are taken from Berrington (1985), Keenan et. al. (1986), and Blum \& Pradhan (1992).

The extinction toward SN 1987A has been discussed in several papers, and there is now general agreement. Walker $\&$ Suntzeff (1990) have measured the reddening in the field of SN $1987 \mathrm{~A}$ by studying the $(U-B)$ and $(B-V)$ colors of 23 early-type stars in the vicinity of the supernova. They determine the reddening to be $E(B-V)_{\text {total }}=0.17 \pm 0.02$. Fitzpatrick \& Walborn (1990) obtained optical and UV spectra of the LMC B0.7 Ia supergiant $\sim 2$ from SN 1987A (see $\S 2$ ). They conclude that $E(B-V)_{\text {total }}=0.16$ for SN 1987A, with Galactic and LMC components of $E(B-V)_{\mathrm{Gal}}=0.06\left(A_{\lambda}\right.$ from Savage \& Mathis 1979) and $E(B-V)_{\text {LMC }}=0.10\left(A_{\lambda}\right.$ for 30 Dor from Fitzpatrick 1985$)$. We adopt the Fitzpatrick \& Walborn extinction toward Sk $-69^{\circ} 203$ for SN 1987A. The total extinction correction factors for the six emission lines are listed in Table 3. In Paper I we adopted $E(B-V)_{\text {total }}=0.20$, the extinction correction for which is also given in Table $3\left[E(B-V)_{\mathrm{Gal}}=0.06\right.$ and $\left.E(B-V)_{\mathrm{LMC}}=0.14\right]$. The line luminosities are computed by correcting for extinction and the distance to the supernova. Assuming isotropic radiation and a distance of $50 \mathrm{kpc}$, the dilution factor is $3.0 \times 10^{47}$.

The ratios depend weakly on the temperature, and in Table 4 we give the resulting values of $\mathrm{N} / \mathrm{C}$ and $\mathrm{N} / \mathrm{O}$ as function of this parameter. In Paper I we argued that $T_{e} \sim 5$

TABLE 3

EXTINCTION CORRECTIONS

\begin{tabular}{ccccc}
\hline \hline Line & $E(B-V)=0.16^{\mathrm{a}}$ & $L_{35}^{\mathrm{b}}$ & $E(B-V)=0.20^{\mathrm{a}}$ & $L_{35}^{\mathrm{b}}$ \\
\hline $\mathrm{N}$ v $\lambda 1240 \ldots \ldots$. & 5.75 & 1.73 & 9.32 & 2.80 \\
$\mathrm{~N}$ IV] $\lambda 1486 \ldots \ldots$ & 4.18 & 1.25 & 6.19 & 1.86 \\
$\mathrm{He}$ III $\lambda 1640 \ldots \ldots$ & 3.76 & 1.13 & 5.34 & 1.60 \\
$\mathrm{O}$ III] $\lambda 1666 \ldots \ldots$ & 3.68 & 1.10 & 5.20 & 1.56 \\
$\mathrm{~N}$ III] $\lambda 1750 \ldots \ldots$ & 3.61 & 1.08 & 5.08 & 1.52 \\
$\mathrm{C}$ III] $\lambda 1908 \ldots \ldots$ & 3.59 & 1.08 & 5.01 & 1.50 \\
\hline
\end{tabular}

\footnotetext{
${ }^{a}$ Extinction correction for observed flux.

${ }^{b}$ Line luminosity in $10^{35} \mathrm{ergs} \mathrm{s}^{-1}$, per unit flux of $1 \times 10^{-13} \mathrm{erg} \mathrm{cm}^{-2}$ $\mathrm{s}^{-1}$, assuming $d=50 \mathrm{kpc}$ and the indicated extinction.
} 
TABLE 4

CNO Abundance Ratios

\begin{tabular}{crccc}
\hline \hline$T_{e} / 10^{4} \mathrm{~K}$ & $\mathrm{~N} / \mathrm{C}$ & Error & $\mathrm{N} / \mathrm{O}$ & Error \\
\hline 1.0 & 15.7 & 2.9 & 1.4 & 0.3 \\
2.0 & 9.4 & 1.7 & 1.5 & 0.4 \\
5.0 & 6.1 & 1.1 & 1.7 & 0.5 \\
10.0 & 4.7 & 0.9 & 1.8 & 0.5
\end{tabular}

$\times 10^{4} \mathrm{~K}$ from [O III] observations (Wampler \& Richichi 1989 ) and the models in LF91. Using this temperature we find that $\mathrm{N} / \mathrm{C}=6.1 \pm 1.1$ and $\mathrm{N} / \mathrm{O}=1.7 \pm 0.5$, where the errors are purely statistical. Accepting an (unrealistically large) uncertainty in $T_{e}$ from $10^{4} \mathrm{~K}$ to $10^{5} \mathrm{~K}$ corresponds to a range in N/C of 4.7-15.7. The statistical errors in the N/C ratio are therefore considerably less than the systematic. Because the lower limit is $\mathrm{N} / \mathrm{C}=4.7$, and a more realistic value is $\mathrm{N} / \mathrm{C}=6.1$, the conclusions in Paper I on the large nitrogen enhancement are unaltered. For comparison, LF96 derive $\mathrm{N} / \mathrm{C}=5.0 \pm 2.0$ and $\mathrm{N} / \mathrm{O}=1.1 \pm 0.4$ from their photoionization/recombination model. The two methods are in good agreement.

There is a weak tendency for the derived $\mathrm{N}$ III/C III ratio to decrease with time. For $400<t<800$ days we find $\mathrm{N}$ iII/ $\mathrm{C}$ III $=5.3 \pm 1.5$ for $T_{e}=5 \times 10^{4} \mathrm{~K}$. The decreasing N III/ C III ratio is consistent with the expectations from the models in LF91 and justifies limiting the abundance analysis to $t \lesssim 500$ days, when $\mathrm{N} / \mathrm{C}=\mathrm{N}$ III/C III is a reasonable approximation.

The nebular analysis has a number of limitations. A nebular model gives a reliable estimate of the ionization structure under steady state conditions or immediately after the gas has been ionized. As the ionizing radiation vanishes, the ionization state evolves away from this structure due to different recombination timescales for the different elements $\left(\mathrm{C}, \mathrm{N}\right.$, and $\mathrm{O}$ ). For example, at $t_{\max }$ the part on the very far side of the ring can be roughly explained by a nebular structure, but the gas on the very near side has had $t_{\max }-t_{\text {rise }}$ days to evolve away from such a structure. Because the observed emission at $t_{\max }$ is an overlay of emission from different parts of the ring which have had different times to evolve off the steady state structure, a pure nebular analysis is bound to have some systematic error. In contrast, LF96 did not have to assume an ionization structure for their models since it and the temperature are calculated in a self-consistent manner. This results in smaller systematic errors related to ionization equilibrium. Given these uncertainties, the CNO abundance ratios in this paper and in LF96 are in good agreement. In the near future spatially resolved spectroscopy of the inner ring with STIS on HST will make possible this type of nebular analysis as a function of position around the ring.

\subsection{Emitting Mass}

From the He II $\lambda 1640$ line we can estimate the mass of the He II emitting gas, i.e., the mass of He III zone. In order to avoid having to compensate for light travel time effects, we evaluate the He II flux near day 400 so that the full volume is observed. Near $t_{\max } \pm 100$ days the observed He II flux was $(9 \pm 2) \times 10^{-14} \mathrm{erg} \mathrm{cm}^{-2} \mathrm{~s}^{-1}$ (estimated uncertainty), and the luminosity was $1.0 \pm 0.2 \times 10^{35}$ ergs $\mathrm{s}^{-1}$, assuming $E(B-V)=0.16$. The He II emissivity does not vary much with time (LF91 and Fig. $6 e$ ), so $L_{\mathrm{He} \mathrm{II}} \approx \int j_{\mathrm{eff}}\left(T_{e}\right) n_{e} n_{\mathrm{He} \text { III }} d V$, where $j_{\text {eff }}$ is the effective emissivity of the $\lambda 1640$ line. In the He III zone $n_{\mathrm{He} \text { III }} \approx n_{\mathrm{He}}$. Furthermore, Wang (1991) finds that $n_{\mathrm{He}} / n_{\mathrm{H}} \approx 0.2$, and we find for the total mass of this zone

$$
\begin{aligned}
M(\mathrm{He} \text { III }) \approx & 1.4 \times 10^{-2}\left(\frac{n_{e}}{2.6 \times 10^{4} \mathrm{~cm}^{-3}}\right)^{-1} \\
& \times\left(\frac{T_{e}}{5 \times 10^{4} \mathrm{~K}}\right)^{0.87} M_{\odot} .
\end{aligned}
$$

This mass estimate only refers to the He III zone. As models by LF96 show, the total mass of ionized gas can be several times larger. A better estimate of the emitting mass is obtained from the $\mathrm{H} \beta$ luminosity. Wampler \& Richichi (1989) estimate $L(\mathrm{H} \beta) \approx 1.4 \times 10^{34} \mathrm{ergs} \mathrm{s}^{-1}$ on day 310 $[E(B-V)=0.16]$. Multiplying this by a factor $3 / 2$, since only $\sim 2 / 3$ of the total ring was within the light echo paraboloid at that time, and again using $n_{\mathrm{He}} / n_{\mathrm{H}} \approx 0.2$, we obtain a total mass of the $\mathrm{H}$ in region

$$
\begin{aligned}
M(\mathrm{He} \text { II }) \approx & 4.7 \times 10^{-2}\left(\frac{n_{e}}{2.6 \times 10^{4} \mathrm{~cm}^{-3}}\right)^{-1} \\
& \times\left(\frac{T_{e}}{5 \times 10^{4} \mathrm{~K}}\right)^{1.02} M_{\odot}
\end{aligned}
$$

on day 395. Because of the high temperature, both the He II line and $\mathrm{H} \beta$ are affected by collisional excitation. The mass estimates are therefore upper limits to the actual masses. (See LF96 for an estimate that takes these model-dependent effects into account.) Our estimates, however, suggest that the He III zone occupies $\sim 30 \%$ of the total ionized region.

The first HST observations of SN 1987A were made on day 1278 (Jakobsen et al. 1991), and we can combine a mass estimate using $\mathrm{H} \beta$ at this time with the observed thickness of the ring to infer the angular extent of the ring, $\Delta \theta$, perpendicular to the ring plane, as seen from the supernova. From the [O II] and [N II] lines the density and temperature of the gas were at this time $n_{e} \sim 1 \times 10^{4} \mathrm{~cm}^{-3}$ and $T_{e} \sim 1.7 \times 10^{4} \mathrm{~K}$ (Wang 1991; Cumming et al. 1997). The flux in $\mathrm{H} \beta$ on day 1280 was $\sim 7.2 \times 10^{-14} \mathrm{erg} \mathrm{cm}^{-2} \mathrm{~s}^{-1}$ (Wang 1991), and the luminosity was $2.2 \times 10^{34} \mathrm{ergs} \mathrm{s}^{-1}$ [for $E(B-V)=0.16]$. The total mass was therefore 4.3 $\times 10^{-2}\left(n_{\mathrm{e}} / 10^{4} \mathrm{~cm}^{-3}\right)^{-1}\left(T_{e} / 1.7 \times 10^{4} \mathrm{~K}\right)^{0.91} M_{\odot}$. Although characteristic of a different density component, this mass is not very different from that near day 400 . Jakobsen et al. (1991) estimate the thickness of the ring in the plane of the sky, $\Delta R_{\perp}$, to $\sim 10 \%$ of the radius, or $\sim 6 \times 10^{16} \mathrm{~cm}$. Plait et al. (1995) found a slightly larger value, $\sim 15 \%$. Because later images obtained shortly after day $\sim 2500$ using the COSTAR corrective optics (Jakobsen et al. 1994) tend to favor the lower estimate of $\sim 10 \%$, we will use this in our analysis. We note, however, that the emitting region could have been somewhat more extended at day 1280 without contradicting the observations by Jakobsen et al. (1994). Assuming a volume filling factor within the ring, $\epsilon$, we get

$$
\begin{aligned}
\Delta \theta \approx & 1.4 \epsilon^{-1}\left(\frac{n_{e}}{1 \times 10^{4} \mathrm{~cm}^{-3}}\right)^{-2} \\
& \times\left(\frac{\Delta R_{\perp} / R}{0.1}\right)^{-1}\left(\frac{T_{e}}{1.7 \times 10^{4} \mathrm{~K}}\right)^{0.91} .
\end{aligned}
$$


This angular extent corresponds to a thickness along the line of sight, $R_{\|}$,

$$
\begin{aligned}
\frac{\Delta R_{\|}}{R} \approx & 0.012 \epsilon^{-1}\left(\frac{n_{e}}{1 \times 10^{4} \mathrm{~cm}^{-3}}\right)^{-2} \\
& \times\left(\frac{\Delta R_{\perp} / R}{0.1}\right)^{-1}\left(\frac{T_{e}}{1.7 \times 10^{4} \mathrm{~K}}\right)^{0.91},
\end{aligned}
$$

around day 1278 , i.e., smaller than the radial extent $(\sim 0.1 R)$, unless $\epsilon \ll 1$. For dynamical reasons we would if anything expect the radial extent to be smaller than the lateral. A solution to this problem may be that the filling factor is considerably smaller than unity, and/or that $\Delta R_{\perp} / R \ll 0.1$. The $H S T$ [O III] images indeed show considerable structure, which may indicate $\epsilon<1$ in the ring. However, it is difficult to see that it can be much less than unity if the ring is resolved in the most recent images (Jakobsen et al. 1994), unless the ring consists of blobs smaller than the $H S T$ resolution, $\sim 0$ ". $1\left(\sim 7.5 \times 10^{16} \mathrm{~cm}\right.$ at a distance of $50 \mathrm{kpc})$. These conclusions agree with those of Plait et al. (1995) and LF96.

\subsection{Density Components}

The line emission becomes dominated by gas of decreasing density because cooling and recombination timescales are inversely proportional to electron density. Therefore, the flux seen at early times is likely to come from gas of considerably higher density than at later times (LF96), consistent with the order-of-magnitude decrease in $n_{e}$ derived in $\S 4.4$ from high-dispersion IUE spectra. This agrees with optical results for [O II] at day 1280 by Wang (1991), who finds $n_{e} \sim 0.7 \times 10^{4} \mathrm{~cm}^{-3}$. A steady decrease in electron density was also found by Plait et al. (1995) when they analyzed HST [O III] images spanning the epoch 12782431 days. The emitting mass may therefore vary with time.

Additional information about the density distribution can be inferred from the low-resolution IUE observations. LF96 find it necessary to include gas with electron densities down to $\sim 6 \times 10^{3} \mathrm{~cm}^{-3}$ to explain the slow decline of the lines after maximum. Such a distribution of densities in the emitting gas is expected from hydrodynamic simulations of the circumstellar structure of SN 1987A (Blondin \& Lundqvist 1993), which show that the highest densities are found close to the ring plane. They also find that there is a large amount of low-density gas both in and out of the plane, possibly accounting for the gas causing the resonance scattering of $\mathrm{N} \mathrm{v} \lambda 1240$. If the low-density gas is ionized up to $\mathrm{N} \mathrm{v}$ and higher, and has a density of $n_{e} \gtrsim \mathrm{a}$ few $\times 10^{2}$ $\mathrm{cm}^{-3}$, this may also give rise to a weak, steady line emission, as predicted by LF91. They argued that such emission should be the sign of the inner parts of the unshocked red supergiant wind. In the interacting winds scenario (e.g., Blondin \& Lundqvist 1993; Martin \& Arnett 1995), the emission could also come from the shocked red supergiant wind (LF96) or perhaps the $\mathrm{H}$ in region interior to the ring (Chevalier \& Dwarkadas 1995). The increasing importance of density components with $n_{e} \lesssim 10^{4} \mathrm{~cm}^{-3}$ after $\sim 1000$ days is consistent with the observed decrease in electron density inferred from density-sensitive line ratios (C III], $\S 4.4$ ) and the optical [O II] and [S II] lines (Wampler et al. 1989; Wang 1991; LF96).

There are also some theoretical indications for gas with $n_{e} \gtrsim 4 \times 10^{4} \mathrm{~cm}^{-3}$. LF96 model three components with dif- ferent densities and find that the bulk of the $\mathrm{C} \mathrm{III]}$ emission at $t \lesssim 400$ days originates in their highest density component $\left(n_{e} \sim 4 \times 10^{4} \mathrm{~cm}^{-3}\right)$, i.e., close to what we derive in $\S 4.4$. It is likely that there is a continuous range of densities rather than components with a few discrete densities. The component with $n_{e} \sim 4 \times 10^{4} \mathrm{~cm}^{-3}$ in LF96 therefore represents a mean value at the high end of this range, indicating the existence of gas with even higher densities. The scatter in the $\mathrm{N} \mathrm{IV}$ ] light curve is considerably larger during the first 400 days than at later times (Fig. 6c), which may be a further sign of components with $n_{e} \gtrsim 4 \times 10^{4} \mathrm{~cm}^{-3}$. Because recombination from $\mathrm{N}$ IV to $\mathrm{N}$ III is rapid, and collisional ionization unimportant, the emissivity of the $\mathrm{N}$ IV] line declines rapidly with time at a given point in the emitting gas (see Fig. 2 of LF91). Therefore, the flux of the $\mathrm{N}$ Iv] line is not severely smoothed by the light echo and responds rapidly to changes in the density of material being swept up by the light echo paraboloid. When higher density gas is encountered, the result is a momentary increase in the observed flux, and this may explain the fluctuations. Fluctuations due to density variations around the ring decrease in importance when the full ring is seen and only recombination is important. The narrow spikes in $\mathrm{N} \mathrm{V}$ and $\mathrm{N} \mathrm{III]}$ between days 350 and 500 (see Fig. 6) are also probably due to higher density gas and the "arcsine" shape of the light curve, characteristic of a ring geometry (e.g., Felten \& Dwek 1991; Lundqvist 1991).

There is also evidence for density structure in the gas after maximum, especially in N III] and N v near day 900 to 1000 . These fluctuations indicate that there may be regions of high density $\sim 2-3$ times further away from the supernova than the ring radius. (This flux increase might also arise from the outer rings, as discussed in $\S$ 4.1.) Several groups have detected blobs in the optical and near-infrared that may be responsible for these fluctuations. Allen et al. (1989) detected a concentration of $\mathrm{He}$ I $1.083 \mu \mathrm{m}$ emission, and they estimate a gas density of $\sim 10^{5} \mathrm{~cm}^{-3}$. Extended $\mathrm{He} \mathrm{I}$ $1.083 \mu \mathrm{m}$ emission was also observed by Elias et al. (1993). Crotts, Kunkel, \& McCarthy (1989) and Hanuschik (1991) detected $\mathrm{H} \alpha$ blobs on days 750 and 950 , respectively, and Cumming \& Meikle (1993) found evidence for a highdensity blob with short lifetime on day 1344 . Cumming \& Meikle estimate that the gas density in the blob has to be $(1-2) \times 10^{5} \mathrm{~cm}^{-3}$ and argue that it may be accounted for by the high-density regions forming off the equatorial plane in the hydrodynamic models by Blondin \& Lundqvist (1993).

\subsection{Comparison With Models}

Comparison of the observations with a realistic, selfconsistent photoionization/recombination model of the circumstellar ring is necessary to test the interpretation of these data. Such a model includes solving the coupled, timedependent equations for temperature and ionization; separating these two aspects leads to an unphysical model. Knowledge of the emissivity as a function of time and position makes it straightforward to incorporate the light echo effect. This was first done by Lundqvist \& Fransson (1987), and later by Fransson \& Lundqvist (1989) and LF91. In those models it was assumed that the emission originated in a spherical shell of density $2.6 \times 10^{4} \mathrm{~cm}^{-3}$. For the ionizing burst at breakout two SN 1987A explosion models were used: the 10L model by Woosley (1988) and the 11E1Y6 model by Shigeyama et al. (1988). Qualitatively, both outburst models reproduced the observed relative line 
strengths surprisingly well, considering the lack of free parameters. The calculations agree fairly well with the rapid increase of the $\mathrm{N}$ III] and $\mathrm{N} v$ lines, and the nearly constant flux in the $\mathrm{N}$ IV] line during the first $\sim 400$ days. The steady He II flux was found to be a result of collisional excitation. The model of LF91 based on the Shigeyama et al. 11E1Y6 spectrum provided the best agreement with both the behavior of the UV lines and the gas temperature, as determined from the [O III] $\lambda \lambda 4363 /(4959+5007)$ ratio. The harder burst spectrum by Shigeyama et al. was therefore favored.

A closer examination, however, reveals several deficiencies. Calculations using both the 10L and 11E1Y6 models produced too rapid an increase in emission flux, especially the $\mathrm{N} v$ line, which rises almost linearly with time (Fig. 6a). As discussed by Lundqvist (1994) and LF96, this may be the result of resonance scattering of the $\mathrm{N} v$ line, not included in the early models. LF96 also find that the slow decline of the $\mathrm{N} v$ line may be a result of resonance scattering in gas external to the ring. The scattering gas could extend as much as $\sim 10^{18} \mathrm{~cm}$ away from the ring plane. The fact that the maxima of the $\mathrm{N} \mathrm{III],} \mathrm{N} \mathrm{IV],} \mathrm{N} \mathrm{v,} \mathrm{and} \mathrm{C} \mathrm{III]} \mathrm{lines}$ are all observed to occur at nearly the same time poses a more serious problem for the LF91 models, which in contrast show the $\mathrm{N}$ III] and $\mathrm{C}$ III] lines peaking considerably later than $\mathrm{N}$ v. In addition, the optical [O III] lines show a persistent emission up to $\sim 1500$ days, in contrast to the early models.

There are two important factors not taken into account in the first models that probably account for these discrepancies. As acknowledged by LF91, the assumption of only one density component is artificial, and the observations clearly indicate that this is not true. A combination of density components, or more realistically a continuum of densities, ranging from $n_{e} \sim 6 \times 10^{3} \mathrm{~cm}^{-3}$ to at least $n_{e} \sim 4$ $\times 10^{4} \mathrm{~cm}^{-3}$, gives considerably better agreement, as demonstrated by LF96. However, such a distribution cannot be specified in a unique way as it depends on the detailed structure of the ring. The other important assumption in LF91 was that of a spherical geometry. A toroidal geometry changes the light curve, when integrating over the light echo paraboloid, as discussed by Lundqvist (1991), Luo (1991), and Dwek \& Felten (1992). A generic feature of a ring model compared to a spherical shell is the rapid rise in the observed fluxes close to maximum light. This basically reflects the amount of emitting gas swept up by the light echo paraboloid per unit time. Even more important, the larger radial thickness required by a toroidal geometry also implies that the ionized gas will be optically thick in the EUV continuum. As already explained, the ionization will then be stratified radially, with several distinct ionization zones (Lundqvist 1992; LF96). The consequence for the light curve is to give nearly simultaneous maxima for the different ions, as is observed. In addition, Ensman \& Burrows (1992) find that the ionizing spectra used in LF91 are probably too soft, as was also pointed out in LF91. Ensman \& Burrows find that the SN spectrum at breakout will have a high-energy tail due to electron scattering.
Ensman \& Burrows also note that light travel time effects smooth the time variation of the spectrum, as may also be the case if the progenitor was nonspherical (see Lundqvist 1992 for a more detailed discussion). However, LF96 find that this effect is unimportant for the ionization of the ring.

In summary, the light curves are fully consistent with a circular ring geometry, both from the rapid rise close to maximum light seen in the $\mathrm{N}$ III] and $\mathrm{N} v$ lines, the turn-on time of these lines, and the near-coincidence of the light curve peaks. A ring geometry also makes it easier to understand the low expansion velocity, $\sim 10 \mathrm{~km} \mathrm{~s}^{-1}$ (Luo \& McCray 1991; Blondin \& Lundqvist 1993). The origin of the ring, however, remains obscure (e.g., Podsiadlowski 1992 for a review). The high $\mathrm{N} / \mathrm{C}$ and $\mathrm{N} / \mathrm{O}$ ratios put strong constraints on the ring, implying that it must have been formed after the dredge-up phase. An interesting proposal by McCray \& Lin (1994) explains the ring as the result of the gradual evaporation of a circumstellar disk around the progenitor. They explain the nitrogen enrichment as a result of gradual spraying of the inner boundary of the disk by the red supergiant wind. Any model for the formation of the ring must also be able to explain the outer rings observed by Wampler et al. (1990), Wang \& Wampler (1992), Burrows et al. (1995), and Plait et al. (1995). The interacting winds model (e.g., Luo \& McCray 1991; Blondin \& Lundqvist 1993) may do so, as demonstrated qualitatively by Martin \& Arnett (1995), and so may the model by Lloyd, O'Brien, \& Kahn (1995). The interacting winds model can also account for the continuum observations of Crotts et al. (1995) (Martin \& Arnett 1995) and the hot gas responsible for the resonance scattering of the $\mathrm{N} v$ line (LF96). However, the high density of the outer rings, a few $10^{3} \mathrm{~cm}^{-3}$ (estimated by LF96) suggests that the interacting winds model needs some adjustment. An interesting extension is that of Chevalier \& Dwarkadas (1995). They assume a lower mass-loss rate during the blue supergiant stage, implying that photoionization by the progenitor is important during the shaping of the nebula. A test of the models for the formation of the observed structure around SN 1987A will be possible as the supernova ejecta strikes the ring shortly after the year $\sim 2000$ (e.g., Masai \& Nomoto 1994; Luo, McCray, \& Slavin 1994; Chevalier \& Dwarkadas 1995; Borkowski, Blondin, \& McCray 1996).

We thank Robert Cumming and Jim Felten for comments on the manuscript and to Martino Romaniello for measuring the positions of stars 2 and 3 in WFPC2 images. G. S. gratefully acknowledges the hospitality and support of the Institut d'Astrophysique de Paris during a 6 month sabbatical leave, during which this paper was completed. This sabbatical was made possible by a NASA Research and Study Fellowship. C. F. and P. L. are both supported by the Swedish Natural Sciences Research Council and the Swedish National Space Board. C. F. is also supported by the Göran Gustafsson Foundation for Research in Natural Sciences and Medicine. This work was supported in part by NASA grant NAS5-2487 to Harvard College Observatory.

\section{REFERENCES}

Aggarwal, K. M., Berrington, K. A., Eissner, W., \& Kingston, A. E. 1986, Recommended Data from the Daresbury Atomic Data Workshop 1985 (Belfast: Daresbury Atomic Data Bank)

Allard, N., Artru, M.-C., Lanz, T., \& Le Dourneuf, M. 1990, A\&AS, 84, 563 Allen, D. A., Meikle, W. P. S., \& Spyromilio, J. 1989, Nature, 342, 403 Ball, L., Campbell-Wilson, D., Crawford, D. F., \& Turtle, A. J. 1995, ApJ,
453, 864

Berrington, K. A. 1985, J. Phys. B, 18, L395

Beuermann, K., Brandt, S., \& Pietsch, W. 1994, A\&A, 281, L45

Bionta, R. M., et al. 1987, Phys. Rev. Lett., 58, 1494

Blinnikov, S. I., \& Nadyozhin, D. K. 1991, in Supernovae, ed. S. E. Woosley (New York: Springer), 213 
Blondin, J. M., \& Lundqvist, P. 1993, ApJ, 405, 337

Blum, R. D., \& Pradhan, A. K. 1992, ApJS, 80, 425

Bohlin, R. C., \& Grillmair, C. J. 1986, ApJS, 66, 209

Bohlin, R. C., \& Holm, A. V. 1980, NASA IUE Newsletter, 10, 37

Borkowski, K., Blondin, J. M., \& McCray, R. 1996, preprint

Burrows, C. J., et al. 1995, ApJ, 454, 680

Cassatella, A., et al. 1987, A\&A, 177, L29 . 1994, A\&A, 281, 594

Chevalier, R. A. 1988, Nature, 332, 514 .1992, Nature, 355, 691

Chevalier, R. A., \& Dwarkadas, V. V. 1995, ApJ, 452, L45

Crotts, A. P. S., \& Heathcote, S. R. 1991, Nature, 350, 683

Crotts, A. P. S., \& Kunkel, W. E. 1991, ApJ, 366, L73

Crotts, A. P. S., Kunkel, W. E., \& Heathcote, S. R. 1995, ApJ, 438, 724

Crotts, A. P. S., Kunkel, W. E., \& McCarthy, P. J. 1989, ApJ, 347, L61

Cumming, R. J. 1994, Ph.D. thesis, Imperial College, London

Cumming, R. J., Lundqvist, P., Meikle, W. P. S., Spyromilio, J., \& Allen,

D. A. 1997 , in preparation

Cumming, R. J., \& Meikle, W. P. S. 1993, MNRAS, 262, 689

Duffy, P., Ball, L., \& Kirk, J. G. 1995, ApJ, 447, 364

Dwek, E., \& Felten, J. E. 1992, ApJ, 387, 551

Elias, J. H., Phillips, M. M., Suntzeff, N. B., Walker, A. R., Gregory, B., \& Depoy, D. L. 1993, in Proc. Massive Stars: Their Lives in the Interstellar Medium, ed. J. P. Cassinelli \& E. B. Churchwell (San Francisco: ASP), 408

Ensman, L., \& Burrows, A. 1992, ApJ, 393, 742

Felten, J. E., \& Dwek, E. 1991, in SN 1987A and Other Supernovae, ed. I. J. Danziger \& K. Kjär (Munich: ESO), 569

Fitzpatrick, E. L. 1985, ApJ, 299, 219

Fitzpatrick, E. L., \& Walborn, N. R. 1990, AJ, 99, 1483

Fleming, J., Hibbert, A., \& Stafford, R. P. 1994, Phys. Scripta, 49, 316

Fransson, C. 1994, in Les Houches, Session LIV 1990, ed. J. Audouze, S. Bludman, R. Mochkovitch, \& J. Zinn-Justin (Holland: Elsevier), 677

Fransson, C., Cassatella, A., Gilmozzi, R., Kirshner, R. P., Panagia, N., Sonneborn, G., \& Wamsteker, W. 1989, ApJ, 336, 429 (Paper I)

Fransson, C., \& Lundqvist, P. 1989, ApJ, 341, L59

Fransson, C., \& Sonneborn, G. 1994, in Frontiers of Space and Groundbased Astronomy, ed. W. Wamsteker, M. S. Longair, \& Y. Kondo (Dordrecht: Kluwer), 249

Garhart, M. P. 1992a, NASA IUE Newsletter, 48, 80

-1992b, NASÁ IUE Newsletter, 48, 98

Gilmozzi, R., et al. 1987, Nature, 328, 318

Gorenstein, P., Hughes, J. P., \& Tucker, W. H. 1994, ApJ, 420, L25

Gould, A. 1994, ApJ, 425, 51 1995, ApJ, 452, 189

Hanuschik, R. W. 1991, A\&A, 217, L21

Hasinger, G., Aschenbach, B., \& Trümper, J. 1996, A\&A, 312, L9

Hauschildt, P. H., Starrfield, S., Austin, S., Wagner, R. M., Shore, S., \& Sonneborn, G. 1994, ApJ, 422, 831

Jakobsen, P., et al. 1991, ApJ, 369, L63

Jakobsen, P., Jedrzejewski, R., Macchetto, F., \& Panagia, N. 1994, ApJ, 435, L47

Kahn, I., \& Duerbeck, H. W. 1991, in SN 1987A and Other Supernovae I. ed. J. Danziger \& K. Kjär (Munich: ESO), 251

Keenan, F. P., Berrington, K. A., Burke, P. G., Dufton, P. L., \& Kingston, A. E. 1986, Phys. Scripta, 34, 216

Keenan, F. P., Feibelman, W., \& Berrington, K. A. 1992, ApJ, 389, 443

Kinney, A. L., Bohlin, R. C., \& Neill, J. D. 1991, PASP, 103, 694

Kirshner, R. P., Sonneborn, G., Crenshaw, D. M., \& Nassiopoulos, G. E. 1987, ApJ, 320, 602

Li, H., \& McCray, R. 1996, ApJ, 456, 370

Lloyd, H. M., O’Brien, T. J., \& Kahn, F. D. 1995, MNRAS, 273, L19

Lucy, L. B. 1987, A\&A, 182, L31

Lundqvist, P. 1991, in SN 1987A and Other Supernovae, ed. I. J. Danziger \& K. Kjär (Munich: ESO), 607 1992, PASP, 104, 787

. 1994, in Circumstellar Media in the Late Stages of Stellar Evolution, ed. R. E. S. Clegg, W. P. S. Meikle, \& I. R. Stevens (Cambridge: Cambridge Univ. Press), 213
Lundqvist, P., \& Fransson, C. 1987, in Proc. ESO Workshop on the SN 1987A, ed. İ. J. Danziger \& K. Kjär (Munich: ESO), 495 1991, ApJ, 380, 575 (LF91) 1996, ApJ, 464, 924 (LF96)

Luo, D. 1991, Ph.D. thesis, Univ. Colorado

Luo, D., \& McCray, R. 1991, ApJ, 379, 659

Luo, D., McCray, R., \& Slavin, J. 1994, ApJ, 430, 264

Martin, C. M., \& Arnett, D. 1995, ApJ, 447, 378

Masai, K., \& Nomoto, K. 1994, ApJ, 424, 924

McCray, R., \& Lin, D. N. C. 1994, Nature, 369, 378

Meikle, W. P. S., Allen, D. A., Spyromilio, J., Cumming, R. J., Varani, G.-F., \& Mobasher, B. 1991, in Proc. ESO/EIPC Workshop, SN 1987A and other Supernovae, ed. I. J. Danziger \& K. Kjär (Munich: ESO), 265 Mendoza, C., \& Zeippen, C. J. 1983, MNRAS, 202, 981

Menzies, J. W. 1991, in Proc. ESO/EIPC Workshop, SN 1987A and Other Supernovae I. J. Danziger \& K. Kjär (Munich: ESO), 209

Munoz Piero, J. R. 1985, ESA IUE Newsletter, 23, 58

Nussbaumer, H., \& Schild, H. 1979, A\&A, 75, L17

Nussbaumer, H., \& Storey, P. J. 1978, A\&A, 64, 139

. 1979, A\&A, 71, L5

Panagia, N., Gilmozzi, R., Macchetto, F., Adorf, H.-M., \& Kirshner, R. P. 1991, ApJ, 380, L23; erratum 386, L31

Plait, P. C., Lundqvist, P., Chevalier, R. A., \& Kirshner, R. P. 1995, ApJ, 439, 730

Podsiadlowski, P. 1992, PASP, 104, 717

Pun, C. S. J., et al. 1995, ApJS, 99, 223

Saio, H., Nomoto, K., \& Kato, M. 1988, Nature, 334, 508

Sanz Fernández de Córdoba, L. 1993, A\&A, 276, 103

Savage, B. D., \& Mathis, J. 1979, ARA\&A, 17, 73

Scuderi, S., Panagia, N., Gilmozzi, R., Challis, P. M., \& Kirshner, R. P. 1996, ApJ, 465, 956

Seaton, M. J. 1987, J. Phys. B. 20, 6431

Shigeyama, T., \& Nomoto, K. 1990, ApJ, 360, 242

Shigeyama, T., Nomoto, K., \& Hashimoto, M. 1988, A\&A, 196, 141

Sonneborn, G. 1991, in Supernovae, Proc. Tenth Santa Cruz Summer

Workshop, ed. S. E. Woosley (New York: Springer), 125

Sonneborn, G., Altner, B., \& Kirshner, R. P. 1987, ApJ, 323, L35

Sonneborn, G., et al. 1990, in Evolution in Astrophysics: IUE Astronomy in the Era of New Space Missions, ESA SP-310

Sonneborn, G., et al. 1988, IAU Circ., 4685

Staveley-Smith, L., et al. 1992, Nature, 355, 147

. 1993, Nature, 366, 136

Thompson, R. W. 1988, NASA IUE Newsletter, 35, 133

Turnrose, B. E., \& Thompson, R. W. 1984, IUE Image Processing Information Manual, Version 2.0 (CSC/TM-84/6058)

Turtle, A. J., et al. 1987, Nature, 327, 38

Walborn, N. R., Lasker, B. M., Laidler, V. G., \& Chu, Y.-H. 1987, ApJ, 321, L41

Walborn, N. R., Phillips, M. M., Walker, A. R., \& Elias, J. H. 1993, PASP, 105,1240

Walker, A. R., \& Suntzeff, N. B. 1990, PASP, 102, 648

Wampler, E. J., \& Richichi, A. 1989, A\&A, 217, 31

Wampler, E. J., Richichi, A., \& Baade, D. 1989, in IAU Colloq. 120, Structures and Dynamics of the Interstellar Medium, ed. G. Tenorio-Tagle, M. Moles, \& J. Melnick (Berlin: Springer), 180

Wampler, E. J., Wang, L., Baade, D., Banse, K., D’Odorico, S., Gouiffes, C., \& Tarenghi, M. 1990, ApJ, 362, L13

Wang, L. 1991, A\&A, 246, L69

Wang, L., \& Wampler, E. J. 1992, A\&A, 262, L9

Weiss, A. 1991, in SN 1987A and Other Supernovae, ed. I. J. Danziger \& K. Kjär (Munich: ESO), 3

West, R. M., Lauberts, A., Jorgensen, H., \& Schuster, H.-E. 1987, A\&A, $177, \mathrm{~L} 1$

Wood, P. R., \& Faulkner, D. J. 1987, IAU Circ., 4739

Woosley, S. E. 1988, ApJ, 330, 218 